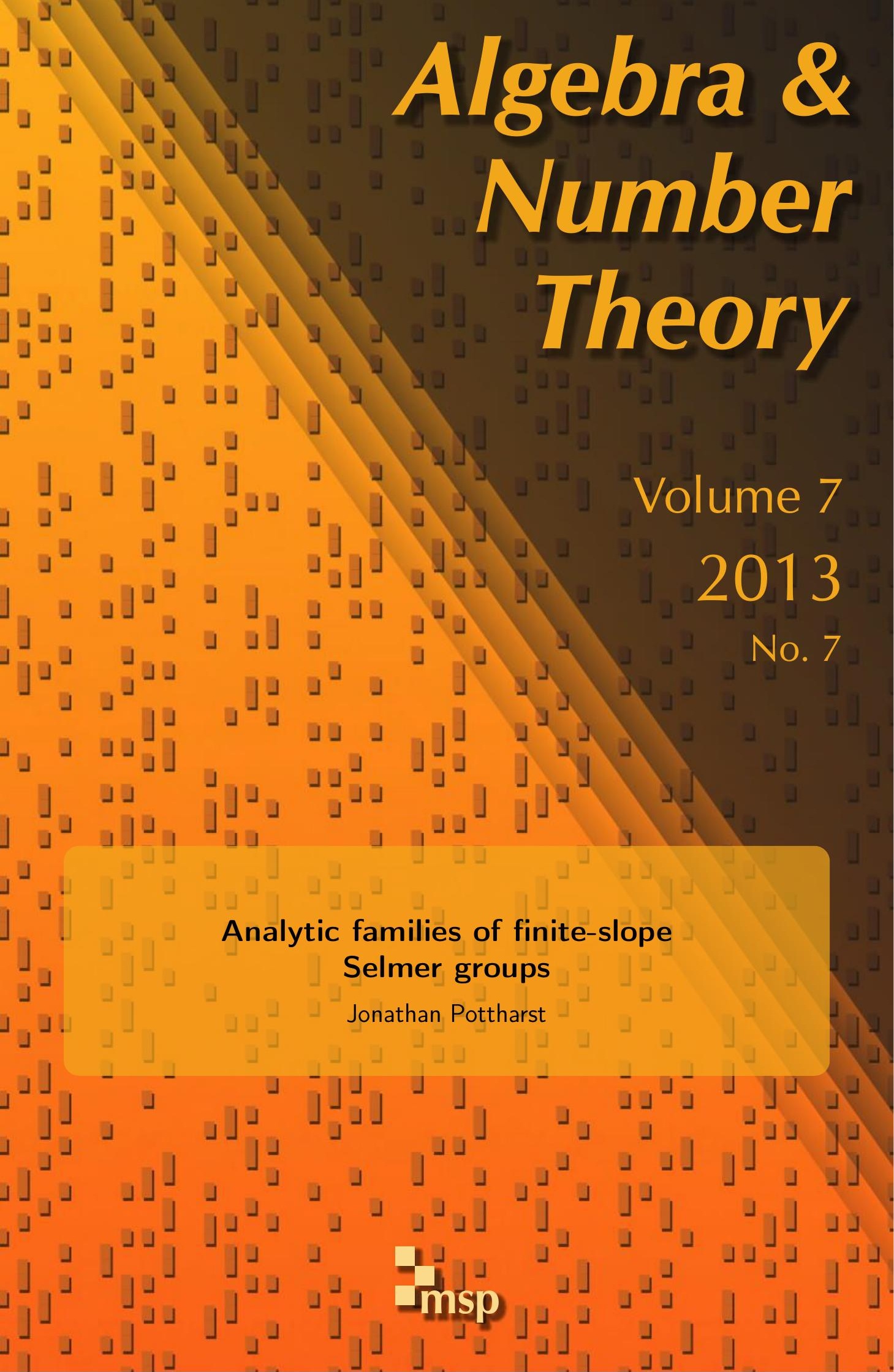




\title{
Analytic families of finite-slope Selmer groups
}

\author{
Jonathan Pottharst
}

\begin{abstract}
We develop a theory of Selmer groups for analytic families of Galois representations, which are only assumed "ordinary" on the level of their underlying $(\varphi, \Gamma)$ modules. Our approach brings the finite-slope nonordinary case of Iwasawa theory onto an equal footing with ordinary cases in which $p$ is inverted.
\end{abstract}

\section{Introduction}

This paper provides new foundations for the algebraic side of Iwasawa theory. We develop a theory of Galois representations and Galois cohomology over $p$-adic analytic spaces. In the classical case, where one works over a complete Noetherian local ring, this amounts to passing to the generic fiber of the associated formal scheme (or, what amounts to the same here, inverting $p$ ). Moreover, we develop a parallel theory for $(\varphi, \Gamma)$-modules varying in families of the same type. The upshot is that we may mimic Greenberg's "ordinary" Iwasawa theory for Galois representations that look ordinary only on the level of their associated $(\varphi, \Gamma)$ module. Although our work was originally motivated by examples coming from eigenvarieties, we have more recently found significant applications even to the classical case of cyclotomic deformations, to be explained in [Pottharst 2012].

This article is rather technical by nature, so we must remain imprecise in the following outline of our results. First, we develop a theory of group cohomology of a profinite group $G$ with coefficients in families of representations over a $p$-adic analytic space $X$ over $\mathbf{Q}_{p}$. By a family of $G$-representations over $X$, we mean a locally finitely generated, flat $\mathrm{O}_{X}$-module $M$, equipped with a continuous map $G \rightarrow \operatorname{Aut}_{\mathscr{O}_{X}}^{\text {cont }}(M)$. In Section 1, we prove the following results:

Theorem. Assume $G$ has finite cohomology on all discrete $G$-modules of finite, $p$ power order, vanishing in degrees greater than e. Then the continuous cohomology with values in $\Gamma(Y, M)$, where $Y \subseteq X$ ranges over affinoid subdomains, gives rise to a perfect complex of coherent $\mathrm{O}_{X}$-modules, vanishing in degrees greater

MSC2010: primary 11R23; secondary 11R34, 12G05.

Keywords: Iwasawa theory, Selmer group, families of Galois representations, $(\varphi, \Gamma)$-modules. 
than $e$. If $f: X^{\prime} \rightarrow X$ is a morphism, then there is a canonical isomorphism $\mathbf{L} f^{*} \mathbf{R} \Gamma_{\text {cont }}(G, M) \stackrel{\sim}{\rightarrow} \mathbf{R} \Gamma_{\text {cont }}\left(G, f^{*} M\right)$.

In the case where $X$ is quasi-Stein, we show that $\Gamma\left(X, \mathbf{R} \Gamma_{\text {cont }}(G, M)\right)$ is computed by $\mathbf{R} \Gamma_{\text {cont }}(G, \Gamma(X, M))$. In the case where $X$ is the generic fiber of the formal scheme $\operatorname{Spf}(A, I)$ and $M$ is the analytification of the $I$-adic $G$-representation $\mathfrak{M}$, we show that $\mathbf{R} \Gamma_{\text {cont }}(G, \mathfrak{M}) \otimes_{A}^{\mathbf{L}} \mathcal{O}_{X} \stackrel{\sim}{\rightarrow} \mathbf{R} \Gamma_{\text {cont }}(G, M)$, thus providing the link with the classical case.

With these tools in hand, it is straightforward to translate into our context the theory of Selmer complexes (and hence Selmer groups) and show that our theory receives the analytification of the classical theory as in the preceding paragraph.

In Section 2, we turn to the case where $G=G_{K}$ is the absolute Galois group of a finite extension $K$ of $\mathbf{Q}_{p}$. We formulate a notion of families of $(\varphi, \Gamma)$-modules over $X$ as above, define their Galois cohomology, and give their basic functorial properties. One important ingredient was conspicuously lacking in a prior version of this paper: we did not know that the $\mathrm{H}^{i}\left(G_{K}, D\right)$ are finitely generated. This has recently been proven; see [Kedlaya et al. 2012]. As for the relation to the cohomology of Galois representations, we prove the following result:

Theorem. There is a functorial isomorphism $\mathbf{R} \Gamma_{\text {cont }}\left(G_{K}, M\right) \stackrel{\sim}{\rightarrow} \mathbf{R} \Gamma\left(G_{K}, \mathbf{D}(M)\right)$, where $\mathbf{D}(M)$ is the family of $\left(\varphi, \Gamma_{K}\right)$-modules associated to $M$.

The essential image of the functor $\mathbf{D}$ is poorly understood at present (see [Hellmann 2012a] for an example of the nontrivial complications that arise), so we note:

Corollary. Let $0 \rightarrow D^{\prime} \rightarrow E \rightarrow D \rightarrow 0$ be a short exact sequence of families of $\left(\varphi, \Gamma_{K}\right)$-modules over $X$ as in the preceding theorem. If $D$ and $D^{\prime}$ arise from families of Galois representations, then so does $E$.

In Section 3, we study the $p$-adic Hodge theory of $\left(\varphi, \Gamma_{K}\right)$-modules, extending to them well-known notions and results for Galois representations. We define ordinary $\left(\varphi, \Gamma_{K}\right)$-modules and formulate the (strict) ordinary local condition in their Galois cohomology and then compare the latter to the Bloch-Kato local conditions. We note that our notion of ordinariness is extremely broad; for example, in the case of modular forms, it includes all cases "of finite slope" (that is, having nonzero $U_{p^{-}}$ eigenvalue up to $p$-stabilization and twisting) or, on the automorphic side, having local Weil-Deligne representation at $p$ that is nonsupercuspidal and of nonscalar Frobenius (the latter condition being conjecturally automatic).

We conclude with a semicontinuity result on the ranks of Selmer groups in an ordinary (in our sense) family, which was also observed by Bellaïche [2012]. Various recent works [Hellmann 2012b; Kedlaya et al. 2012; Liu 2012] show that ordinary families are abundant: in particular, any family that is refined in the sense of Bellaïche and Chenevier [2009], such as an eigenvariety, is automatically ordinary away from a proper Zariski-closed subset. Thus, our hypotheses are not very restrictive. 
Background. The practice of $p$-adically interpolating Selmer groups goes back to the seminal work of Mazur [1972], where abelian varieties at good ordinary primes are treated; it is suggested there that "the situation is remarkably different" for nonordinary primes. That one definitively cannot integrally interpolate the usual Selmer groups in a naïve way was confirmed by work of Schneider [1987]. For motives satisfying an "ordinary" hypothesis, Greenberg [1989; 1994a] found a purely Galoiscohomological replacement for the $p$-adic Hodge-theoretic local conditions that is amenable to interpolation. The latter approach was axiomatized by Nekováŕ [2006].

The above left open the question of what happens in the nonordinary setting. Work of Amice and Vélu [1975] and Višik [1976] showed that the analytic $p$-adic L-functions of modular forms belong to $\mathrm{O}_{X}$, and not to $\Lambda[1 / p]$ as in the ordinary case, where $\Lambda$ is the Iwasawa algebra and $X$ is the generic fiber of $\operatorname{Spf}(\Lambda, \mathfrak{m})$. Then, heavily using Fontaine's tools of $p$-adic Hodge theory, Perrin-Riou [1994b; 2000] constructed algebraic $p$-adic L-functions (i.e., would-be characteristic ideals), also belonging to $\Lambda_{\infty}$. Somewhat surprisingly, her construction eschewed the Selmer groups with finer local conditions although it recovered their characteristic ideals in the ordinary case. Using her language, in the case of modular forms, Kato [2004] used his Euler system to prove a divisibility in an Iwasawa main conjecture and in the ordinary case deduced a statement about Selmer groups.

The next advance came when Kisin [2003] made a Galois-theoretic study of the eigencurve, identifying the relevant two-dimensional $p$-adic Galois representations as those admitting a crystalline period after twist. Colmez [2005; 2008; 2010] followed the analogy between these representations and principal series, reformulated Kisin's condition in terms of $(\varphi, \Gamma)$-modules (terming it "trianguline"), and made a rigorous $p$-adic local Langlands correspondence for them. These two works have influenced, e.g., Bellaïche and Chenevier [2009], who refine the methods to make a detailed study of Selmer groups in the infinitesimal neighborhoods of classical points on eigenvarieties.

We briefly mention that somewhat recently there has been progress in nonordinary cyclotomic Iwasawa theory employing similar tools to ours but resulting in mysteriously different outputs. The theory was initiated by R. Pollack and S. Kobayashi (building on work of M. Kurihara) and generalized by F. Sprung, A. Lei, D. Loeffler, and S. Zerbes. See [Pottharst 2012] for references and more commentary on this direction.

Future directions. Our theory is incomplete in that we have direct access to no integral information, having chosen to exchange it for major simplifications in $p$ adic Hodge theory when working only up-to-isogeny. The remedy for this is likely to be the use of Euler-Poincaré formulas to construct integral isogeny invariants, following [Bloch and Kato 1990; Fontaine and Perrin-Riou 1994; Perrin-Riou 2000]. Still, the theory has several applications. It essentially subsumes Perrin-Riou's 
cyclotomic Iwasawa theory, as explained in [Pottharst 2012]. Nekováŕ's work on the parity of Selmer groups in families, as well as the parity conjecture for ordinary Hilbert modular forms of parallel weight, readily generalizes to our setting. In joint work with K. S. Kedlaya, L. Xiao, and the author [Kedlaya et al. 2012], a perfectness and duality result for the Galois cohomology of families of $\left(\varphi, \Gamma_{K}\right)$-modules is applied to give a general construction of triangulations of eigenvarieties as well as a classification of rank-one families of $\left(\varphi, \Gamma_{K}\right)$-modules; for other recent progress on triangulations, see [Chenevier 2010; Hellmann 2012b; Kedlaya et al. 2012; Liu 2012]. Bellaïche has also used our Selmer groups to prove an Iwasawa main conjecture for Eisenstein series using their nonordinary choice of $p$-stabilization (personal communication). Finally, Benois [2011; 2009] has used methods similar to ours to study $\mathscr{L}$-invariants of Perrin-Riou's Iwasawa L-functions.

This paper is intended as the first step of an Iwasawa theory within the $p$-adic Langlands program. Namely, Galois-theoretic eigenvarieties for reductive groups $H$ over $\mathbf{Q}$ should be moduli of ordinary filtrations on the $(\varphi, \Gamma)$-modules of universal Galois deformations with values in ${ }^{\mathrm{L}} H$. For each $\iota:{ }^{\mathrm{L}} H \rightarrow \mathrm{GL}_{d}$ preserving the ordinariness of the filtration, the Galois-theoretic eigenvariety will then have a natural Selmer module. First steps in this direction have been made by Chenevier [2010] and Hellmann [2010; 2012b]. The automorphic (i.e., usual) eigenvariety associated to $H$ will map to the Galois-theoretic one by virtue of its family of Galois representations. A generalization à la Kisin of the ordinary " $R=T$ " conjectures would predict this map to be an isomorphism, and Iwasawa theory would relate the $\iota$ Selmer module to the $p$-adic L-function interpolating the $\iota$-L-values of automorphic representations on $H$.

Notation. Throughout, we fix a prime $p$ and a finite extension $E$ of $\mathbf{Q}_{p}$ with ring of integers $\mathrm{O}_{E}$.

Let $a \leq b$ be integers. For $* \in\{[a, b], \mathrm{b},+,-, \varnothing\}$, we say that a complex or graded module is $*$-bounded if it is, respectively, concentrated in degrees $[a, b]$, bounded, bounded above, bounded below, or is arbitrary.

If $\left(Z, O_{Z}\right)$ denotes a ringed topos and $? \in\{\varnothing, \mathrm{ft}\}$, we write $\mathbf{K}_{?}^{*}(Z)$ for the category of complexes of $\mathrm{O}_{Z}$-modules, each of whose cohomologies is $*$-bounded and, if $?=\mathrm{ft}$, satisfies a finiteness condition to be made precise as it arises. We write $\mathbf{D}_{?}^{*}(Z)$ for its derived category, and we write $\mathbf{G r}_{\text {? }}^{*}(Z)$ for the category of $*$-bounded graded $\mathrm{O}_{Z}$-modules, each of whose components satisfies ?. Denote by $[\cdot]: \mathbf{K}_{?}^{*}(Z) \rightarrow \mathbf{D}_{?}^{*}(Z)$ and $\mathrm{H}^{*}: \mathbf{D}_{?}^{*}(Z) \rightarrow \mathbf{G r}_{?}^{*}(Z)$ the obvious functors. Denote also $\mathbf{D}_{\text {perf }}^{[a, b]}(Z) \subseteq \mathbf{D}_{\mathrm{ft}}^{[a, b]}(Z)$ the strictly full subcategory consisting of objects $X$ quasi-isomorphic to complexes $C^{\bullet}$ concentrated in degrees $[a, b]$ consisting of $\mathrm{ft}$ and flat modules. On the latter category, $X \mapsto X^{*}=\mathbf{R} \operatorname{Hom}_{C_{Z}}\left(X, O_{Z}\right)$ is in each case under consideration an anti-involution, and for $X \cong\left[C^{\bullet}\right]$ as above, $X^{*}$ is represented by $\operatorname{Hom}_{\mathrm{O}_{Z}}\left(C^{\bullet}, \mathrm{O}_{Z}\right)$. 


\section{Group cohomology}

1A. Continuous cochains: local calculations. We will say that an $\mathbb{O}_{E}$-module $M$ is linearly topologized if it is equipped with a topology with basis around the identity consisting of a decreasing sequence $M_{n}$ of $\mathrm{O}_{E}$-submodules. We will say that an $\mathscr{O}_{E}$-algebra $R$ is linearly topologized (as an algebra) if the system of submodules $R_{n}$ can be chosen so that $R_{n} \cdot R_{n} \subseteq R_{n}$. For such $R$, we will say that an $R$-module $M$ is linearly topologized compatibly with $R$ if the systems $R_{n}$ and $M_{n}$ of open submodules can be chosen so that $R_{n} \cdot M_{n} \subseteq M_{n}$; in particular, the multiplication map $R \times M \rightarrow M$ is bicontinuous.

Let $G$ be a profinite group. A continuous $G$-module is a linearly topologized $O_{E^{-}}$ module $M$ endowed with a continuous map $G \rightarrow \operatorname{Aut}_{\mathscr{O}_{E}}^{\text {cont }}(M)$, the latter equipped with the compact-open topology. Given a linearly topologized $O_{E}$-algebra $R$, a continuous $R[G]$-module is an $R$-module $M$ that is linearly topologized compatibly with $R$ endowed with a continuous map $G \rightarrow \operatorname{Aut}_{R}^{\text {cont }}(M)$, the latter again equipped with the compact-open topology. We define the complex $\mathrm{C}_{\mathrm{cont}}^{\bullet}(G, M) \in \mathbf{K}^{+}(R)$ of continuous cochains on $G$ with values in $M$ to be $\mathrm{C}_{\text {cont }}^{i}(G, M)=\operatorname{Map}^{\text {cont }}\left(G^{i}, M\right)$ with the usual differential (see, e.g., [Nekovár 2006, 3.4.1.2]). We denote its image in $\mathbf{D}^{+}(R)$ by $\mathbf{R} \Gamma_{\text {cont }}(G, M)$ and its cohomology by $\mathrm{H}_{\text {cont }}^{*}(G, M) \in \mathbf{G r}^{+}(R)$. The latter defines a functor that, of course, turns short exact sequences into long exact sequences provided the usual existence of continuous (though not necessarily grouptheoretic) sections. The reader may check that, under our hypotheses below, one always has the necessary continuous sections for turning short exact sequences into long exact sequences.

In order to get reasonable behavior, we will need to impose some hypotheses. The following are sufficient for our applications:

Hypotheses A. (1) $G$ is a profinite group having finite $p$-cohomological dimension $e$, and $\# \mathrm{H}_{\text {cont }}^{i}(G, T)<\infty$ for all finite discrete $\mathbf{F}_{p}[G]$-modules $T$ and all $i \geq 0$.

(2) $A$ is a Noetherian $O_{E}$-algebra, separated and complete with respect to a proper ideal $I$ containing a power of $p$ and equipped with the $I$-adic topology.

(3) $M$ is a finite-type $A$-module, considered with its $I$-adic topology and equipped with a continuous $A[G]$-module structure.

(4) The $A$-module $M$ is flat.

We say that an $A$-module satisfies the condition " $\mathrm{ft}$ " if it is of finite type.

Note that, under Hypotheses A(1)-(3), since $A / I^{N}$ has the discrete topology for $N>0$, the stabilizer of any element of $M / I^{N}$ is open in $G$; since $M$ is finitely generated, we see that $G$ acts on $M / I^{N}$ through a finite quotient.

The following is the main result of this section: 
Theorem 1.1. Assume Hypotheses A.

(1) The complexes $\mathrm{C}_{\mathrm{cont}}^{\bullet}\left(G, M / I^{N}\right)$ and $\mathrm{C}_{\mathrm{cont}}^{\bullet}(G, M)$ consist of flat $A / I^{N}$-modules and A-modules, respectively.

(2) The inverse system $\left\{\mathrm{H}_{\text {cont }}^{*}\left(G, M / I^{N}\right)\right\}_{N}$ satisfies Mittag-Leffler.

(3) The natural map $\mathrm{H}_{\text {cont }}^{*}(G, M) \rightarrow \lim _{N} \mathrm{H}_{\text {cont }}^{*}\left(G, M / I^{N}\right)$ is an isomorphism.

(4) The $\mathrm{H}_{\mathrm{cont}}^{i}(G, M)$ are finitely generated A-modules and vanish for $i>e$.

The above theorem shows that $\mathrm{C}_{\mathrm{cont}}^{\bullet}(G, M) \in \mathbf{K}_{\mathrm{ft}}^{[0, e]}(A)$. In fact, copying the proof of [Nekovár 2006, 4.2.9] verbatim (in the case $a=b=0, \mathscr{S}=\{1\}$ ), one obtains the following strengthening:

Corollary 1.2. Assume Hypotheses A. Then $\mathbf{R} \Gamma_{\text {cont }}(G, M) \in \mathbf{D}_{\mathrm{perf}}^{[0, e]}(A)$.

Lemma 1.3. Assume Hypotheses A(1)-(3).

(1) For any compact topological space $X$, the natural maps

$$
\operatorname{Map}^{\text {cont }}(X, M) / I^{N} \rightarrow \operatorname{Map}^{\text {cont }}\left(X, M / I^{N}\right)
$$

are isomorphisms.

(2) The natural maps

$$
\mathrm{C}_{\mathrm{cont}}^{\bullet}(G, M) / I^{N} \rightarrow \mathrm{C}_{\mathrm{cont}}^{\bullet}\left(G, M / I^{N}\right)
$$

are isomorphisms of complexes.

(3) One has $\mathrm{H}_{\mathrm{cont}}^{i}(G, M)=0$ for $i>e$.

(4) If $M$ is annihilated by a power of $I$, then $\mathrm{H}_{\mathrm{cont}}^{*}(G, M)$ is a finitely generated A-module.

(5) If Hypothesis (4) holds too, then for each $N>0$ the complexes $\mathrm{C}_{\text {cont }}^{\bullet}\left(G, M / I^{N}\right)$ and $\mathrm{C}_{\mathrm{cont}}^{\bullet}(G, M)$ consist of flat $A / I^{N}$-modules and A-modules, respectively.

Proof. For (1), the main fact we will use is that the continuous maps from a compact topological space to a discrete topological space are precisely the locally constant ones.

The map of the claim is surjective by the discreteness of $M / I^{N}$; that it is injective amounts to the claim that the natural map $I^{N} \cdot \operatorname{Map}^{\operatorname{cont}}(X, M) \rightarrow \operatorname{Map}^{\text {cont }}\left(X, I^{N} M\right)$ is surjective. As the source of this map is complete and $I^{N} M \stackrel{\sim}{\rightarrow} \lim _{k} I^{N} M / I^{N+k} M$, it suffices to show that the maps $\left.I^{N} \cdot \operatorname{Map}^{\text {cont }}(X, M) \rightarrow \operatorname{Map}^{\text {cont }} \overleftarrow{(X}^{k} I^{N} M / I^{N+k} M\right)$ for $k \geq 0$ are surjective. But the $I^{N} M / I^{N+k} M$ are discrete, so the latter claim is obvious.

For (2), the maps are clearly compatible with differentials, so we must check that they are isomorphisms term-by-term. For the $i$-th term, this results from (1) with $X=G^{i}$. 
To prove (3), observe that by the universal property of the inverse limit one has $\mathrm{C}_{\text {cont }}^{\bullet}(G, M)=\lim _{N} \mathrm{C}_{\text {cont }}^{\bullet}\left(G, M / I^{N}\right)$ with surjective transition maps; hence, for each $i$, we have short exact sequences

$$
0 \rightarrow \mathbf{R}^{1} \underset{N}{\lim } \mathrm{H}_{\text {cont }}^{i-1}\left(G, M / I^{N}\right) \rightarrow \mathrm{H}_{\text {cont }}^{i}(G, M) \rightarrow \underset{N}{\lim } \mathrm{H}_{\text {cont }}^{i}\left(G, M / I^{N}\right) \rightarrow 0
$$

Since each $M / I^{N}$ is a discrete $p$-primary $G$-module, the claim for $i>e+1$ follows. For $i=e+1$, the inverse system $\left\{\mathrm{H}_{\text {cont }}^{i-1}\left(G, M / I^{N}\right)\right\}_{N}$ has surjective maps because each $\mathrm{H}_{\text {cont }}^{i}\left(G, I^{N} M / I^{N+k} M\right)=0$. This implies that the $\mathbf{R}^{1} \lim _{N}$-term vanishes, giving the claim in this case too.

Next we treat (4). We may perform a dévissage to reduce to the case where $(I, p) M=0$, and then we choose an open normal subgroup $H \subseteq G$ with $M^{H}=M$. Since $M$ is $\mathbf{F}_{p}$-flat, the natural map $\mathrm{H}_{\text {cont }}^{*}\left(H, \mathbf{F}_{p}\right) \otimes_{\mathbf{F}_{p}} M \rightarrow \mathrm{H}_{\text {cont }}^{*}(H, M)$ is an isomorphism. By Hypothesis (1), the term $\mathrm{H}_{\text {cont }}^{*}\left(H, \mathbf{F}_{p}\right)$ is finite; hence, $\mathrm{H}_{\text {cont }}^{*}(H, M)$ is finitely generated over $A$. Now consider the spectral sequence

$$
\mathrm{H}_{\text {cont }}^{i}\left(G / H, \mathrm{H}_{\text {cont }}^{j}(H, M)\right) \Longrightarrow \mathrm{H}_{\text {cont }}^{i+j}(G, M) \text {. }
$$

The terms on the left are finitely generated over $A$ because $G / H$ is finite. This forces $\mathrm{H}_{\text {cont }}^{*}(G, M)$ to be finitely generated.

Finally, for (5), it follows from (2) that for any ideal $J$ of $A$ one has

$$
J \otimes_{A} \mathrm{C}_{\text {cont }}^{\bullet}(G, M) \stackrel{\sim}{\rightarrow} J \otimes_{A} \underset{N}{\lim _{N}} \mathrm{C}_{\text {cont }}^{\bullet}(G, M) / I^{N}
$$

But $A$ is Noetherian, so $J$ is finitely presented, and $\otimes_{A} J$ commutes with taking inverse limits of surjective systems. Therefore, one has

$$
J \otimes_{A} \mathrm{C}_{\text {cont }}^{\bullet}(G, M) \stackrel{\sim}{\rightarrow} \underset{N}{\lim }\left(J \otimes_{A} \mathrm{C}_{\text {cont }}^{\bullet}(G, M)\right) / I^{N},
$$

and the right-hand side is clearly $I$-adically separated. This verifies that $\mathrm{C}_{\text {cont }}^{\bullet}(G, M)$ is " $I$-adically ideal separated". Hence, by the well-known local criterion for flatness, e.g., [Matsumura 1989, Theorem 22.3(1, 5)], this complex consists of flat $A$-modules if and only if each of its respective reductions modulo $I^{N}$ is $A / I^{N}$-flat. But since $M$ is $A$-flat, it follows that the $M / I^{N}$ are $A / I^{N}$-flat, and indeed so are the complexes $\mathrm{C}_{\text {cont }}^{\bullet}\left(G, M / I^{N}\right)=\lim _{H \unlhd G \text { open }}\left(M / I^{N}\right)^{\oplus(G / H)^{\bullet}}$. We conclude by again using (2). $\square$ Proof of Theorem 1.1. The claim (1) follows immediately from Lemma 1.3(5), and the last part of the claim (4) is Lemma 1.3(3).

The remaining claims follow from the results of [Berthelot and Ogus 1978, Appendix B], whose conventions for handling inverse limits in the derived category we now recall. Namely, we consider the poset $\mathbf{N}$ of nonnegative integers as a Grothendieck site with the discrete topology: only identity maps are coverings. A sheaf on $\mathbf{N}$ is merely an inverse system, and the condition that a sheaf be flasque 
simply amounts to the Mittag-Leffler condition. We equip $\mathbf{N}$ with the sheaf $A$. of rings given by $N \mapsto A_{N}=A / I^{N+1}$ and work in the derived category $\mathbf{D}(\mathbf{N}, A$.) of A.-modules on $\mathbf{N}$.

Write $M_{N}=M \otimes_{A} A_{N}$; the $\mathrm{C}_{\text {cont }}^{i}\left(G, M_{N}\right)$ determine a complex $C$. of sheaves of $A$--modules. Lemma 1.3(3) shows that $\left[C^{\bullet}\right] \in \mathbf{D}^{-}(\mathbf{N}, A$.), and Lemma 1.3(5) implies that $\left[C_{N+1}^{\bullet}\right] \otimes_{A_{N+1}}^{\mathbf{L}} A_{N}$ is represented by the complex $C_{N+1}^{\bullet} \otimes_{A_{N+1}} A_{N}$, which by Lemma 1.3(2) is isomorphic to $C_{N}^{\bullet}$. The latter two claims mean that $C^{\bullet}$ is what in [Berthelot and Ogus 1978] is called a quasiconsistent complex. Lemma 1.3(4) shows (in particular) that $\left[C_{0}^{\bullet}\right] \in \mathbf{D}_{\mathrm{ft}}^{-}\left(A_{0}\right)$, and hence, the main finiteness result [Berthelot and Ogus 1978, Proposition B.7 $]^{1}$ applies to $C^{\bullet}$. and $\mathbf{R} \lim _{N}\left[C^{\bullet}\right]$. Finally, Lemma 1.3(2) shows that $C^{\bullet}$ is a complex of flasque sheaves, and therefore, $\mathbf{R} \lim _{N}\left[C^{\bullet}\right]=\left[\lim _{N} C_{N}^{\bullet}\right]=\left[\mathrm{C}_{\text {cont }}^{\bullet}(G, M)\right]$, allowing us to rephrase the finiteness result as claims (2)-(4).

We turn to base-changing properties. We say that $B$ is an $I$-adic A-algebra if it is a Noetherian $A$-algebra that is $(I B)$-adically separated and complete and equipped with the $(I B)$-adic topology; when no confusion may arise, we abusively denote by $I$ the ideal $I B$ of $B$.

Theorem 1.4. Assume Hypotheses A hold, and let B be an I-adic A-algebra.

(1) The natural map

$$
\mathbf{R} \Gamma_{\text {cont }}(G, M) \otimes_{A}^{\mathbf{L}} B \rightarrow \mathbf{R} \Gamma_{\text {cont }}\left(G, M \otimes_{A} B\right)
$$

is an isomorphism in $\mathbf{D}_{\text {perf }}^{[0, e]}(B)$.

(2) There is a canonical spectral sequence

$$
\mathrm{E}_{2}^{i j}=\operatorname{Tor}_{-i}^{A}\left(\mathrm{H}_{\text {cont }}^{j}(G, M), B\right) \Longrightarrow \mathrm{H}_{\text {cont }}^{i+j}\left(G, M \otimes_{A} B\right) .
$$

(3) If $B$ is flat over A or becomes flat after inverting $p$, then the natural map

$$
\mathrm{H}_{\text {cont }}^{*}(G, M) \otimes_{A} B \rightarrow \mathrm{H}_{\text {cont }}^{*}\left(G, M \otimes_{A} B\right)
$$

is an isomorphism or becomes an isomorphism after inverting p, respectively.

Lemma 1.5. Assume Hypotheses A hold, and let B be an I-adic A-algebra.

(1) For any compact topological space $X$, the natural map

$$
\operatorname{Map}^{\text {cont }}(X, M) \widehat{\otimes}_{A} B \rightarrow \operatorname{Map}^{\text {cont }}\left(X, M \widehat{\otimes}_{A} B\right)
$$

is an isomorphism.

${ }^{1}$ In the statement of this result, the hypothesis " $D_{0} \in \mathbf{D}_{\mathrm{ft}}^{-}\left(\mathbf{N}, A\right.$.)" should read " $D_{0} \in \mathbf{D}_{\mathrm{ft}}^{-}\left(A_{0}\right)$ ". 
(2) The natural map

$$
\mathrm{C}_{\text {cont }}^{\bullet}(G, M) \widehat{\otimes}_{A} B \rightarrow \mathrm{C}_{\text {cont }}^{\bullet}\left(G, M \otimes_{A} B\right)
$$

is an isomorphism of complexes.

(3) The natural map

$$
\mathrm{C}_{\text {cont }}^{\bullet}(G, M) \otimes_{A} B \rightarrow \mathrm{C}_{\text {cont }}^{\bullet}(G, M) \widehat{\otimes}_{A} B
$$

is a quasi-isomorphism.

Proof. We treat (1). One has natural maps

$$
\begin{aligned}
\operatorname{Map}^{\text {cont }}(X, M) \otimes_{A} B / I^{N} & =\operatorname{Map}^{\text {cont }}(X, M) / I^{N} \otimes_{A} B / I^{N} \\
& \stackrel{\alpha}{\rightarrow} \operatorname{Map}^{\operatorname{cont}}\left(X, M / I^{N}\right) \otimes_{A} B / I^{N} \\
& \stackrel{\beta}{\rightarrow} \operatorname{Map}^{\operatorname{cont}}\left(X, M \otimes_{A} B / I^{N}\right) .
\end{aligned}
$$

The map $\alpha$ is an isomorphism by Lemma 1.3(2). One easily deduces that $\beta$ is an isomorphism from the fact that all of $M / I^{N}, B / I^{N}$, and $M \otimes_{A} B / I^{N}$ are discrete. Thus, we deduce the claim by passing to the inverse limit over $N$.

For (2), the map is clearly compatible with differentials, so we must check that it is an isomorphism term-by-term. For the $i$-th term, this results from applying (1) with $X=G^{i}$ and noting that $M \widehat{\otimes}_{A} B=M \otimes_{A} B$ because $M$ is finitely generated over $A$.

We now show (3). Choose a quasi-isomorphism $D^{\bullet} \rightarrow \mathrm{C}_{\text {cont }}^{\bullet}(G, M) \otimes_{A} B$ with $D^{\bullet}$ a bounded-above complex of finitely generated, flat $B$-modules. Because both $D^{\bullet}$ and $\mathrm{C}_{\text {cont }}^{\bullet}(G, M) \otimes_{A} B$ are $B$-flat, the induced maps

$$
D^{\bullet} \otimes_{B} B / I^{N} \rightarrow \mathrm{C}_{\text {cont }}^{\bullet}(G, M) \otimes_{A} B / I^{N}
$$

are quasi-isomorphisms. On the other hand, both systems satisfy Mittag-Leffler so that, applying $\lim _{N}$, the induced map $\widehat{D}^{\bullet} \rightarrow \mathrm{C}_{\text {cont }}^{\bullet}(G, M) \widehat{\otimes}_{A} B$ is also a quasiisomorphism. We conclude by tracing around the commutative diagram

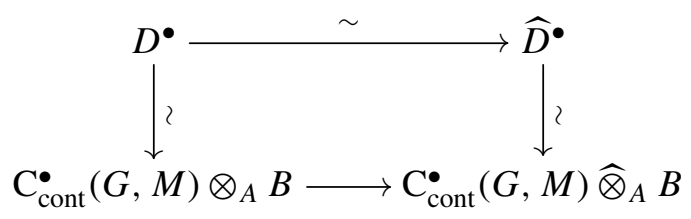

where the top horizontal isomorphism is because the $D^{i}$ are finitely generated.

Proof of Theorem 1.4. The claim (1) follows from Lemmas 1.3(5) and 1.5(2)-(3), and (2) follows from (1) formally. To see (3), consider the spectral sequence of (2). After inverting $p$ if necessary, all terms with $i \neq 0$ vanish, yielding degeneracy at $\mathrm{E}_{2}$, whence the desired isomorphism.

Often, we are only given a finitely generated $A[1 / p]$-module $M$ equipped with the structure of a continuous $A[1 / p][G]$-module. If there exists a finitely generated, 
flat, $G$-stable $A$-submodule $M_{0} \subseteq M$ such that $M_{0}[1 / p]=M$, then $M_{0} \otimes_{A} B$ plays the same role inside $M \otimes_{A[1 / p]} B[1 / p]$, and the preceding finiteness, perfectness, and base-change results apply with $A[1 / p], B[1 / p]$, and $M$ in place of $A, B$, and $M_{0}$, respectively, and all objects occurring in it are independent of the choice of $M_{0}$.

For any strictly $E$-affinoid space $Y$, we write $\mathscr{A}_{Y}$ for the set of unit balls of Banach algebra norms on $\Gamma\left(Y, O_{Y}\right)$. Any $A \in \mathscr{A}_{Y}$, equipped with $I=(p)$, satisfies Hypothesis (2). If $M$ is a finitely generated, flat $\Gamma\left(Y, O_{Y}\right)$-module equipped with the structure of a continuous $\Gamma\left(Y, O_{Y}\right)[G]$-module, then by [Chenevier 2009, Lemme 3.18] one has $M=M_{0}[1 / p]$ for $M_{0}$ a finitely generated, flat, $G$-stable $A$-submodule, for some $A \in \mathscr{A}_{Y}$, and $\mathbf{R} \Gamma(G, M)=\mathbf{R} \Gamma\left(G, M_{0}\right)[1 / p]$ belongs to $\mathbf{D}_{\text {perf }}^{[0, e]}\left(\Gamma\left(Y, \mathcal{O}_{Y}\right)\right)$. If $f: Y^{\prime} \rightarrow Y$ is a morphism of affinoid spaces, then the image of $A$ in $\Gamma\left(Y^{\prime}, O_{Y^{\prime}}\right)$ is contained in some $B \in A_{Y^{\prime}}$, and any such $B$ is a $p$-adic $A$-algebra. Thus, by the preceding paragraph, we may apply Theorem 1.4 with $A$ and $B$ replaced by $\Gamma\left(Y, O_{Y}\right)$ and $\Gamma\left(Y^{\prime}, O_{Y^{\prime}}\right)$. In particular, if $Y^{\prime}$ is an affinoid subdomain of $Y$, then $B[1 / p]=\Gamma\left(Y^{\prime}, O_{Y^{\prime}}\right)$ is flat over $A[1 / p]=\Gamma\left(Y, O_{Y}\right)$, so Theorem 1.4(3) applies.

1B. General p-adic analytic spaces. Let $X$ be a $p$-adic analytic space over $E$, and let $U$ be an admissible affinoid covering that is quasiclosed under intersection, meaning that whenever $Y, Y^{\prime} \in \mathcal{U}$ then $Y \cap Y^{\prime}$ has an admissible cover consisting of elements of $\mathcal{U}$. We consider $\mathcal{U}$ as a poset category and equip it with the discrete Grothendieck topology: only identity maps are coverings, and all presheaves are sheaves. Thus, homological algebra of sheaves on $u$ is essentially carried out independently over each affinoid. It is a ringed site via the rule $\Gamma\left(Y, \mathrm{O}_{u}\right)=\Gamma\left(Y, \mathrm{O}_{Y}\right)$, and an $\mathrm{O}_{u}$-module $M$ consists of the data of a $\Gamma\left(Y, \mathscr{O}_{Y}\right)$-module $\Gamma(Y, M)$ for each $Y \in \mathcal{U}$ together with a morphism $\Gamma(Y, M) \rightarrow \Gamma\left(Y^{\prime}, M\right)$ of $\Gamma\left(Y, O_{Y}\right)$-modules for each $Y, Y^{\prime} \in \mathcal{U}$ with $Y^{\prime}$ contained in $Y$, satisfying the obvious compatibility law for $Y^{\prime \prime} \subseteq Y^{\prime} \subseteq Y$. Similarly to the situation in [Berthelot and Ogus 1978, Appendix B], we say that a complex $C^{\bullet}$ of $\mathrm{O}_{u}$-modules is quasiconsistent if each induced map

$$
\Gamma\left(Y, C^{\bullet}\right) \otimes_{\Gamma\left(Y, O_{Y}\right)} \Gamma\left(Y^{\prime}, \mathscr{O}_{Y^{\prime}}\right) \rightarrow \Gamma\left(Y^{\prime}, C^{\bullet}\right)
$$

is a quasi-isomorphism. Since $\Gamma\left(Y^{\prime}, O_{Y^{\prime}}\right)$ is $\Gamma\left(Y, O_{Y}\right)$-flat, we in fact have an isomorphism

$$
\left[\Gamma\left(Y, C^{\bullet}\right)\right] \otimes_{\Gamma\left(Y, \mathscr{O}_{Y}\right)}^{\mathbf{L}} \Gamma\left(Y^{\prime}, \mathscr{O}_{Y^{\prime}}\right) \stackrel{\sim}{\rightarrow}\left[\Gamma\left(Y^{\prime}, C^{\bullet}\right)\right]
$$

in $\mathbf{D}\left(\Gamma\left(Y^{\prime}, \mathscr{O}_{Y^{\prime}}\right)\right)$. We say that a quasiconsistent complex of $\mathcal{O}_{u}$-modules $C^{\bullet}$ is of finite type, or satisfies the condition "ft", or is flat if, for all $i \in \mathbf{Z}$ and for all $Y \in \mathcal{U}$, the $\Gamma\left(Y, O_{Y}\right)$-module $\Gamma\left(Y, C^{i}\right)$ is of finite type or flat, respectively. Quasiconsistent $\mathrm{O}_{u}$-modules form an abelian subcategory of all $\mathrm{O}_{u}$-modules that is closed under extensions. Note that a complex of quasiconsistent $\mathrm{O}_{\imath}$-modules is a quasiconsistent complex of $\mathrm{O}_{u}$-modules but not in general conversely. 
From now on, by a complex of $\mathrm{O}_{\mathrm{u}}$-modules, we implicitly mean quasiconsistent complex of $\mathrm{O}_{u}$-modules unless said otherwise. Especially, for $* \in\{+,-, \mathrm{b}, \varnothing\}$, the notations $\mathbf{K}_{\mathrm{ft}}^{*}(u), \mathbf{D}_{\mathrm{ft}}^{*}(u), \mathbf{D}_{\text {perf }}^{[a, b]}(\mathcal{U})$, and $\mathbf{G r}_{\mathrm{ft}}^{*}(u)$ denote categories of quasiconsistent complexes or graded modules of finite type. We have the obvious commutative diagram, where the vertical arrows denote taking sections over $Y \in U$ :

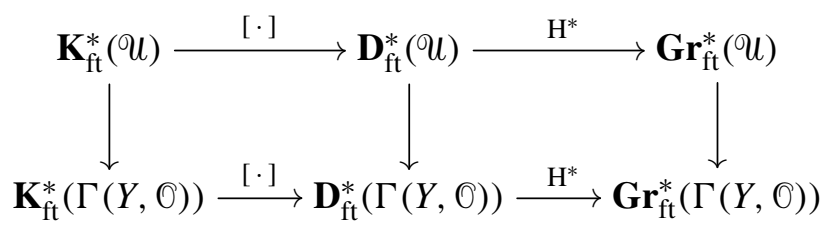

By a family of $G$-representations over $X$, we mean a locally finitely generated, flat $O_{X}$-module $M$, equipped with a continuous map $G \rightarrow \operatorname{Aut}_{\mathscr{O}_{X}}^{\text {cont }}(M)$. Then $M$ determines by restriction a finitely generated, flat $\mathrm{O}_{u}$-module, which we also denote by $M$, whose group of sections over each $Y \in \mathcal{U}$ is a continuous $\Gamma(Y, \mathcal{O})[G]$-module. By the discussion at the end of Section 1A, it follows from Theorem 1.4 that the $0_{u}$-module determined by the rule $Y \mapsto \mathrm{C}_{\text {cont }}^{\bullet}(G, \Gamma(Y, M))$ is quasiconsistent; combined with Theorem 1.1(4), this rule hence determines an object of $\mathbf{K}_{\mathrm{ft}}^{[0, e]}(\mathcal{U})$, which we denote by $\mathrm{C}_{\text {cont }}^{\bullet}(G, M)$. Its class $\mathbf{R} \Gamma_{\text {cont }}(G, M)$ in the derived category belongs to $\mathbf{D}_{\text {perf }}^{[0, e]}(u)$, and its cohomology $\mathrm{H}_{\text {cont }}^{*}(G, M)$ belongs to $\mathbf{G r}_{\mathrm{ft}}^{[0, e]}(\mathcal{U})$. For any $Y \in U$, we have

$$
\Gamma\left(Y, \mathrm{C}_{\text {cont }}^{\bullet}(G, M)\right)=\mathrm{C}_{\text {cont }}^{\bullet}(G, \Gamma(Y, M)),
$$

and it follows from the commutativity of the diagram (1-1) that also

$$
\begin{aligned}
\Gamma\left(Y, \mathbf{R} \Gamma_{\text {cont }}(G, M)\right) & =\mathbf{R} \Gamma_{\text {cont }}(G, \Gamma(Y, M)), \\
\Gamma\left(Y, \mathrm{H}_{\text {cont }}^{*}(G, M)\right) & =\mathrm{H}_{\text {cont }}^{*}(G, \Gamma(Y, M)),
\end{aligned}
$$

so all we really have is a compatible family of cohomology data over the affinoids in question. Using Kiehl's theorem, we may identify $\mathrm{H}_{\text {cont }}^{*}(G, M)$ to an object of $\mathbf{G} \mathbf{r}_{\mathrm{coh}}^{[0, e]}(X)$, in which the subscript denotes coherent $\mathrm{O}_{X}$-modules. Since the latter is invariant under passing between $U$ and a refinement, we canonically associate to $M$ a coherent analytic sheaf on $X$ whose sections over any affinoid domain $Y$ give the continuous cohomology of $G$ with coefficients in the sections of $M$ over $Y$.

We now state a general base-change theorem for group cohomology. Suppose we are given a morphism $f: X^{\prime} \rightarrow X$ of $p$-adic analytic spaces over $E$, and let $u^{\prime}$ be an admissible affinoid covering of $X^{\prime}$ that is quasiclosed under intersection with the property that for each $Y^{\prime} \in \mathcal{U}^{\prime}$ there exists $Y \in \mathcal{U}$ with $f\left(Y^{\prime}\right) \subseteq Y$. Any object $C^{\bullet}$ of $\mathbf{K}_{\mathrm{ft}}^{-}(U)$ gives rise to an object $\mathbf{L} f^{*} C^{\bullet}$ of $\mathbf{D}_{\mathrm{ft}}^{-}\left(U^{\prime}\right)$ by the usual recipe.

Theorem 1.6. (1) The natural map

$$
\mathbf{L} f^{*} \mathbf{R} \Gamma_{\text {cont }}(G, M) \rightarrow \mathbf{R} \Gamma_{\text {cont }}\left(G, f^{*} M\right)
$$


is an isomorphism in $\mathbf{D}_{\text {perf }}^{[0, e]}\left(\mathcal{U}^{\prime}\right)$.

(2) There exists a spectral sequence in coherent $\mathrm{O}_{X^{\prime}}$-modules

$$
\mathrm{E}_{2}^{i j}=\operatorname{Tor}_{-i}^{f^{-1} \mathscr{O}_{X}}\left(f^{-1} \mathrm{H}_{\mathrm{cont}}^{j}(G, M), \mathscr{O}_{X^{\prime}}\right) \Longrightarrow \mathrm{H}_{\mathrm{cont}}^{i+j}\left(G, f^{*} M\right) .
$$

(3) If $f$ is flat, then the natural map

$$
f^{*} \mathrm{H}_{\text {cont }}^{*}(G, M) \rightarrow \mathrm{H}_{\text {cont }}^{*}\left(G, f^{*} M\right)
$$

is an isomorphism in $\mathbf{G r}_{\text {coh }}^{[0, e]}\left(X^{\prime}\right)$.

Proof. After clearing away the abstract nonsense using (1-2), this is just an application of Theorem 1.4 to $A[1 / p]=\Gamma\left(Y, O_{Y}\right)$ and $B[1 / p]=\Gamma\left(Y^{\prime}, O_{Y^{\prime}}\right)$ for each pair of affinoids $Y \in \mathcal{U}$ and $Y^{\prime} \in \mathcal{U}^{\prime}$ with $f\left(Y^{\prime}\right) \subseteq Y$, using Kiehl's theorem to patch back up.

1C. Quasi-Stein spaces. Continuing with $X$ and $M$ as in Section 1B, assume that $X$ is quasi-Stein: it admits an admissible covering $U$ by an increasing union of strictly $E$-affinoid subdomains $Y_{1} \subseteq Y_{2} \subseteq \cdots$, each of whose restriction maps $\Gamma\left(Y_{n+1}, \mathcal{O}_{Y_{n+1}}\right) \rightarrow \Gamma\left(Y_{n}, \mathcal{O}_{Y_{n}}\right)$ has dense image.

Let $M_{n}$ be the $\Gamma\left(Y_{n}, \mathcal{O}_{Y_{n}}\right)$-module $\Gamma\left(Y_{n}, M\right)$. The ring $A_{\infty}=\lim _{n} \Gamma\left(Y_{n}, O_{Y_{n}}\right)=$ $\Gamma\left(X, O_{X}\right)$ is a commutative Fréchet-Stein algebra, and $M_{\infty}=\lim _{n} M_{n}=\Gamma(X, M)$ is a coadmissible $A_{\infty}$-module in the sense of [Schneider and Teitelbaum 2003, §3]. "Theorem A" for such modules states that the natural maps $M_{\infty} \rightarrow M_{n}$ have dense image; they induce isomorphisms $M_{\infty} \otimes_{A_{\infty}} \Gamma\left(Y_{n}, \mathcal{O}_{Y_{n}}\right) \stackrel{\sim}{\rightarrow} M_{n}$. Also, this denseness suffices for Mittag-Leffler considerations, whence "Theorem B" states that for all $i>0$ one has $\mathbf{R}^{i} \lim _{n} M_{n}=0$. We obtain an exact equivalence between coherent sheaves on $X$ and coadmissible modules over $A_{\infty}$. In particular, the subcategory of all $A_{\infty}$-modules consisting of the coadmissible ones forms an abelian subcategory that is closed under extensions. We say that an $A_{\infty}$-module satisfies condition " $\mathrm{ft}$ " if its coadmissible.

Turning to cohomology, each of the maps $\mathrm{C}_{\text {cont }}^{\bullet}\left(G, M_{n+1}\right) \rightarrow \mathrm{C}_{\text {cont }}^{\bullet}\left(G, M_{n}\right)$ has dense image, and $\mathrm{C}_{\text {cont }}^{\bullet}\left(G, M_{\infty}\right)=\lim _{n} \mathrm{C}_{\text {cont }}^{\bullet}\left(G, M_{n}\right)$ by the definition of the inverse limit, so Mittag-Leffler gives $\mathrm{H}_{\text {cont }}^{*}\left(G, M_{\infty}\right)=\lim _{n} \mathrm{H}_{\text {cont }}^{*}\left(G, M_{n}\right)$. Hence, $\mathrm{C}_{\text {cont }}^{*}\left(G, M_{\infty}\right)$ is an object of $\mathbf{K}_{\mathrm{ft}}^{[0, e]}\left(A_{\infty}\right)$, where the subscript means that the cohomology modules are required to be coadmissible.

The following theorem follows easily from the preceding discussion:

Theorem 1.7. The natural maps

$$
\begin{aligned}
\mathbf{R} \Gamma_{\text {cont }}\left(G, M_{\infty}\right) & \rightarrow \mathbf{R} \underset{n}{\lim }\left[\mathrm{C}_{\text {cont }}^{\bullet}\left(G, M_{n}\right)\right], \\
\mathrm{H}_{\text {cont }}^{*}\left(G, M_{\infty}\right) & \rightarrow \underset{n}{\lim } \mathrm{H}_{\text {cont }}^{*}\left(G, M_{n}\right), \\
\mathrm{H}_{\text {cont }}^{*}\left(G, M_{\infty}\right) \otimes_{A_{\infty}} \Gamma\left(Y_{n}, \mathcal{O}\right) & \rightarrow \mathrm{H}_{\text {cont }}^{*}\left(G, M_{n}\right)
\end{aligned}
$$


are isomorphisms.

Remark 1.8. The usual explicit construction shows that, for any $M$ and $M^{\prime}$ and $n \leq \infty$, the group cohomology $\mathrm{H}_{\text {cont }}^{1}\left(G, \operatorname{Hom}_{\Gamma\left(Y_{n}, 0\right)}\left(M_{n}, M_{n}^{\prime}\right)\right)$ is in canonical bijection with the Yoneda group $\operatorname{Ext}_{\Gamma\left(Y_{n}, \mathcal{O}\right)[G] \text {-cont }}^{1}\left(M_{n}, M_{n}^{\prime}\right)$ of extensions of $M_{n}^{\prime}$ by $M_{n}$ in the category of continuous $\Gamma\left(Y_{n}, \mathcal{O}\right)[G]$-modules.

1D. Generic fibers of formal spectra. In this section, we let $A$ and $M$ satisfy Hypotheses A. Assume for this discussion that $A / I$ is a finitely generated $O_{E^{-}}$ algebra and that $A$ is $p$-torsion-free. We now compare the group cohomology of $M$ to that of its generic fiber.

Let $A_{n}^{0}=A\left[I^{n} / p\right]$, the $A$-subalgebra of $A[1 / p]$ generated by all $i / p$ with $i \in I^{n}$, and let $A_{n}$ be its $p$-adic completion. Each $A_{n}[1 / p]$ is a strictly $E$-affinoid algebra, and maps $A_{n+1}[1 / p] \rightarrow A_{n}[1 / p]$ arising from the inclusions $A_{n+1}^{0} \subseteq A_{n}^{0}$ correspond to inclusions $Y_{n} \subseteq Y_{n+1}$ of affinoid subdomains. The increasing system $Y_{1} \subseteq Y_{2} \subseteq \ldots$ forms an admissible affinoid covering of its union $X$. (See [de Jong 1995, §7.1] for details.) It is clear that $X$ is a quasi-Stein space so that Section $1 \mathrm{C}$ applies.

The powers of the ideals $p A_{n}^{0}, I A_{n}^{0} \subseteq A_{n}^{0}$ are cofinal so that $A_{n}$ is also the $I$-adic completion of $A_{n}^{0}$. In particular, each $A_{n}$ is an $I$-adic $A$-algebra. Each $A_{n}[1 / p]$ is flat over $A[1 / p]$. The following base-changing theorem now follows easily from the preceding work:

Theorem 1.9. For $n \leq \infty$, the natural maps

$$
\begin{aligned}
\mathbf{R} \Gamma_{\text {cont }}(G, M) \otimes_{A}^{\mathbf{L}} A_{n} & \rightarrow \mathbf{R} \Gamma_{\text {cont }}\left(G, M \otimes_{A} A_{n}\right), \\
\mathrm{H}_{\text {cont }}^{*}(G, M) \otimes_{A} A_{n}[1 / p] & \rightarrow \mathrm{H}_{\text {cont }}^{*}\left(G, M \otimes_{A} A_{n}[1 / p]\right)
\end{aligned}
$$

are isomorphisms.

Remark 1.10. By Remark 1.8 and Theorem 1.9, for $n \leq \infty$, the map on Yoneda groups $\operatorname{Ext}_{A[G] \text {-cont }}^{1}\left(M, M^{\prime}\right) \rightarrow \operatorname{Ext}_{A_{n}[1 / p][G] \text {-cont }}\left(M \otimes_{A} A_{n}[1 / p], M^{\prime} \otimes_{A} A_{n}[1 / p]\right)$ determined by applying $\otimes_{A} A_{n}[1 / p]$ to an extension class $0 \rightarrow M^{\prime} \rightarrow E \rightarrow M \rightarrow 0$ induces an isomorphism

$\operatorname{Ext}_{A[G]-\text { cont }}^{1}\left(M, M^{\prime}\right) \otimes_{A} A_{n} \stackrel{\sim}{\rightarrow} \operatorname{Ext}_{A_{n}[1 / p][G]-c o n t}\left(M \otimes_{A} A_{n}[1 / p], M^{\prime} \otimes_{A} A_{n}[1 / p]\right)$.

Morally, $A[1 / p] \subseteq A_{\infty}$ consists of the $p$-adically bounded functions on $X$, so calling the image of $\operatorname{Ext}_{A[G] \text {-cont }}^{1}\left(M, M^{\prime}\right)[1 / p]$ in $\operatorname{Ext}_{A_{\infty}[G] \text {-cont }}^{1}\left(M \otimes_{A} A_{\infty}, M^{\prime} \otimes_{A} A_{\infty}\right)$ the bounded extension classes is reasonable.

1E. Selmer complexes. We now copy ideas of Nekovár [2006] into the context of the preceding sections.

In the preceding sections, we describe a variety of situations all of the following sort: one is given a profinite group $G$ satisfying Hypothesis (1), a ringed topos $Z$ built from $p$-adic rings, and a locally finitely generated flat $O_{Z}$-module $M$ with 
continuous $\mathrm{O}_{Z}[G]$-module structure. In each situation, we show that the continuous cohomology objects satisfy $\mathrm{C}_{\text {cont }}^{\bullet}(G, M) \in \mathbf{K}_{\mathrm{ft}}^{[0, e]}(Z), \mathbf{R} \Gamma_{\text {cont }}(G, M) \in \mathbf{D}_{\text {perf }}^{[0, e]}(Z)$, and $\mathrm{H}_{\text {cont }}^{*}(G, M) \in \mathbf{G r}_{\mathrm{ft}}^{[0, e]}(Z)$ and that their formation commutes with (derived) pullback along appropriate morphisms of topoi $f: Z^{\prime} \rightarrow Z$ and in a certain case pushforward as well. Specifically, we treat the following cases, using a tilde to denote the associated ringed topos:

(1) $(A, I)$ and $M$ satisfy Hypotheses $\mathrm{A}(2)-(4)$, and $B$ is an $I$-adic $A$-algebra. We define $Z=(\operatorname{Spec} A)^{\sim}$ and $Z^{\prime}=(\operatorname{Spec} B)^{\sim}$, and let $f$ be the induced morphism.

(2) $M=M_{0}[1 / p]$ where $(A, I)$ and $M_{0}$ satisfy Hypotheses $\mathrm{A}(2)-(4)$, and $B$ is an $I$-adic $A$-algebra. We define $Z=(\operatorname{Spec} A[1 / p])^{\sim}$ and $Z^{\prime}=(\operatorname{Spec} B[1 / p])^{\sim}$, and let $f$ be the induced morphism.

(3) $X$ is a $p$-adic analytic space over $E, M$ is a family of $G$-representations over $X$, $U$ is an admissible affinoid covering of $X$ that is quasiclosed under intersection, $f_{0}: X^{\prime} \rightarrow X$ is a morphism of $p$-adic analytic spaces over $E$, and $U^{\prime}$ is an admissible affinoid covering of $X^{\prime}$ that is quasiclosed under intersection with the property that for each $Y^{\prime} \in \mathcal{U}^{\prime}$ there exists $Y \in \mathcal{U}$ with $f_{0}\left(Y^{\prime}\right) \subseteq Y$. We define $Z=\left(u, \sigma_{u}\right)^{\sim}$ and $Z^{\prime}=\left(u^{\prime}, O_{u^{\prime}}\right)^{\sim}$, and let $f=\tilde{f}_{0}$.

(4) $X$ is a quasi-Stein $p$-adic analytic space over $E, M$ is a family of $G$-representations over $X$, and $U=\left\{Y_{n}\right\}_{n \geq 1}$ is an increasing admissible affinoid covering as in Section 1C. We define $Z=\left(\operatorname{Spec} \Gamma\left(X, \mathcal{O}_{X}\right)\right)^{\sim}$ and $Z^{\prime}=\left(\mathcal{U}, \mathcal{O}_{u}\right)^{\sim}$, and let $f$ be the induced morphism, interpreting "finite type" over $Z$ to mean "coadmissible". In this case, the formation of cohomology also commutes with (derived) pushforward along $f$.

(5) $(A, I)$ and $M$ satisfy Hypotheses $\mathrm{A}(2)-(4)$, and moreover, $A$ is $p$-torsion-free and $A / I$ is a finitely generated $O_{E}$-algebra. We define $Z=(\operatorname{Spec} A)^{\sim}$, the rings $A_{n}$ for $n \leq \infty$ as in Section $1 \mathrm{D}$, and $Z^{\prime}=\left(\operatorname{Spec} A_{n}[1 / p]\right)^{\sim}$, and let $f$ be the induced morphism, in the case $n=\infty$ interpreting "finite type" over $Z^{\prime}$ to mean "coadmissible".

In this section, we specialize to the case of the group $G=G_{K, S}$ defined in the next paragraph and give analogous results in each of these scenarios where the continuous cohomology objects have been replaced by Selmer complexes relative to appropriate local conditions. We also give variants of Tate's local and Poitou-Tate's global arithmetic duality theorems for these objects.

We let $K$ be a finite extension of $\mathbf{Q}$, and we let $S$ be a finite set of finite places of $K$ containing all $v$ dividing $p$. We choose a maximal algebraic extension $K_{S}$ of $K$ unramified outside $S \cup\{\infty\}$, and put $G_{K, S}=\operatorname{Gal}\left(K_{S} / K\right)$. We also choose, for each $v \in S$, an algebraic closure $K_{v}^{\text {alg }}$ of $K_{v}$ together with a $K$-algebra embedding $K_{S} \hookrightarrow K_{v}^{\text {alg }}$, and put $G_{v}=\operatorname{Gal}\left(K_{v}^{\mathrm{alg}} / K_{v}\right)$. Denote by $I_{v} \subset G_{v}$ the inertia subgroup. 
We write res $v$ for the map $G_{v} \rightarrow G_{K, S}$ given by restriction along our chosen embedding as well as for the map $\mathrm{C}_{\text {cont }}^{\bullet}\left(G_{K, S}, M\right) \rightarrow \mathrm{C}_{\text {cont }}^{\bullet}\left(G_{v}, M\right)$ it induces, via pullback, on cocycles. Assume for simplicity that $p \neq 2$ or $K$ is totally complex. By arithmetic duality theory, the groups $G_{K, S}$ and $G_{v}$ for $v \in S$ satisfy Hypothesis (1) with $e=2$ (see [Neukirch et al. 2008, 8.3.10, 8.3.17-19, and 7.1.8]). In the exceptional case where $p=2$ and $K$ is not totally complex, one can get a similar theory working with a little more care; see [Nekovarr 2006, 5.7], and note that the complication is annihilated by inverting 2 anyway.

We place ourselves in one of the scenarios (1)-(5) above, where the hypotheses are made relative to the group $G=G_{K, S}$. By a (*-bounded) local condition $\Delta_{v}$ at $v \in S$, we mean the data of an object $U_{v}^{\bullet} \in \mathbf{K}_{\mathrm{ft}}^{*}(Z)$ and a morphism $i_{v}: U_{v}^{\bullet} \rightarrow \mathrm{C}_{\text {cont }}^{\bullet}\left(G_{v}, M\right)$. Assume we are given the data $\Delta=\left\{\Delta_{v}\right\}_{v \in S}$ of a local condition $\Delta_{v}$ for each $v \in S$. We define the Selmer complex $\widetilde{\mathrm{C}}_{\mathrm{f}}^{\bullet}\left(G_{K, S}, M ; \Delta\right)$ of $M$ with respect to $\Delta$ to be the complex

$$
\text { Cone }\left[\mathrm{C}_{\text {cont }}^{\bullet}\left(G_{K, S}, M\right) \oplus \bigoplus_{v \in S} U_{v} \stackrel{\oplus_{v \in S}\left(\operatorname{res}_{v}-i_{v}\right)}{\longrightarrow} \bigoplus_{v \in S} \mathrm{C}_{\text {cont }}^{\bullet}\left(G_{v}, M\right)\right][-1] .
$$

We denote by $\mathbf{R} \widetilde{\Gamma}_{\mathrm{f}}\left(G_{K, S}, M ; \Delta\right)$ the image of the Selmer complex in the derived category, and we denote its cohomology groups, which we call the (extended) Selmer groups, by $\widetilde{\mathrm{H}}_{\mathrm{f}}^{*}\left(G_{K, S}, M ; \Delta\right)$. For brevity, we usually suppress the dependence on $\Delta$ from the notation. By the definition of the extended Selmer groups in terms of a mapping cone, one has an exact triangle

$$
\mathbf{R} \widetilde{\Gamma}_{\mathrm{f}}\left(G_{K, S}, M ; \Delta\right) \rightarrow \mathbf{R} \Gamma_{\text {cont }}\left(G_{K, S}, M\right) \rightarrow \bigoplus_{v \in S} E_{v},
$$

where the objects $E_{v}=\operatorname{Cone}\left(i_{v}\right)$ sit in exact triangles

$$
U_{v} \stackrel{i_{v}}{\rightarrow} \mathbf{R} \Gamma_{\text {cont }}\left(G_{v}, M\right) \stackrel{j_{v}}{\rightarrow} E_{v}
$$

Thus, the image of the extended Selmer group in $\mathrm{H}_{\text {cont }}^{i}\left(G_{K, S}, M\right)$ consists of those classes that everywhere locally live in the image of the $\mathrm{H}^{i}\left(i_{v}\right)$; this image is what one more traditionally encounters in the literature, so we call it the nonextended Selmer group $\mathrm{H}_{\mathrm{f}}^{i}\left(G_{K, S}, M ; \Delta\right)$.

The following finiteness theorem is just an application to each of $G=G_{K, S}$ and $G=G_{v}$ of the finiteness theorems of the preceding sections in light of the exact triangles (1-3) and (1-4):

Theorem 1.11. The complex $\widetilde{\mathrm{C}}_{\mathrm{f}}^{\bullet}\left(G_{K, S}, M ; \Delta\right)$ belongs to $\mathbf{K}_{\mathrm{ft}}^{*}(Z)$. In particular, $\mathbf{R} \widetilde{\Gamma}_{\mathrm{f}}\left(G_{K, S}, M ; \Delta\right) \in \mathbf{D}_{\mathrm{ft}}^{*}(Z)$ and $\widetilde{\mathrm{H}}_{\mathrm{f}}^{*}\left(G_{K, S}, M ; \Delta\right) \in \mathbf{G r}_{\mathrm{ft}}^{*}(Z)$. If for each $v \in S$ one has $\left[U_{v}\right] \in \mathbf{D}_{\text {perf }}^{[0,2]}(Z)$, then $\mathbf{R} \widetilde{\Gamma}_{\mathrm{f}}\left(G_{K, S}, M ; \Delta\right)$ belongs to $\mathbf{D}_{\text {perf }}^{[0,3]}(Z)$. 
We turn to treat base change. If the local conditions are bounded above (up to quasi-isomorphism), on the one hand we may form $\mathbf{L} f^{*} \widetilde{\mathrm{C}}_{\mathrm{f}}^{\bullet}\left(G_{K, S}, M ; \Delta\right)$. On the other hand, for $v \in S$, we may form the local condition $f^{*} \Delta_{v}$ for $f^{*} M$ by choosing a representative in $\mathbf{K}_{\mathrm{ft}}^{-}\left(Z^{\prime}\right)$ of the morphism

$$
\mathbf{L} f^{*} U_{v} \stackrel{\mathbf{L} f^{*} i_{v}}{\longrightarrow} \mathbf{L} f^{*} \mathbf{R} \Gamma_{\text {cont }}\left(G_{v}, M\right) \stackrel{\sim}{\rightarrow} \mathbf{R} \Gamma_{\text {cont }}\left(G_{v}, f^{*} M\right),
$$

and we write $f^{*} \Delta=\left\{f^{*} \Delta_{v}\right\}_{v \in S}$. The following theorem is similarly deduced from the finiteness and base-changing theorems of the preceding sections:

Theorem 1.12. In the situations (1)-(5) above, assume the local conditions $\Delta$ are bounded above. Then the natural map

$$
\mathbf{L} f^{*} \mathbf{R} \widetilde{\Gamma}_{\mathrm{f}}\left(G_{K, S}, M ; \Delta\right) \rightarrow \mathbf{R} \widetilde{\Gamma}_{\mathrm{f}}\left(G_{K, S}, f^{*} M ; f^{*} \Delta\right)
$$

is an isomorphism in $\mathbf{D}_{\mathrm{ft}}^{-}\left(Z^{\prime}\right)$.

If for each $v \in S$ one has $\left[U_{v}\right] \in \mathbf{D}_{\text {perf }}^{[0,2]}(Z)$, then the isomorphism takes place in $\mathbf{D}_{\text {perf }}^{[0,3]}\left(Z^{\prime}\right)$.

In the case (4) above, so $X$ is quasi-Stein with increasing admissible affinoid covering $U=\left\{Y_{n}\right\}_{n \geq 1}$, there is also a pushforward result. Assume we are given, instead of local conditions on the global sections $\Gamma(X, M)$ as in the preceding theorem, local conditions on $\Delta^{\prime}$ on $M$ considered as a sheaf on $U$. Thus, we have a quasiconsistent family of morphisms $\Gamma\left(Y_{n}, U_{v}^{\prime \bullet}\right) \rightarrow \Gamma\left(Y_{n}, \mathrm{C}_{\text {cont }}^{\bullet}(G, M)\right)$ for varying $n$. Assume that the maps $\Gamma\left(Y_{n+1}, U_{v}^{\prime \bullet}\right) \rightarrow \Gamma\left(Y_{n}, U_{v}^{\prime \bullet}\right)$ have dense image. We form local conditions $\Delta$ for $\Gamma(X, M)$ using the morphisms

$$
i_{v}^{\bullet}: U_{v}^{\bullet}=\lim _{n} \Gamma\left(Y_{n}, U_{v}^{\prime \bullet}\right) \rightarrow \underset{n}{\lim } \Gamma\left(Y_{n}, \mathrm{C}_{\text {cont }}^{\bullet}\left(G_{v}, M\right)\right)=\mathrm{C}_{\text {cont }}^{\bullet}\left(G_{v}, \Gamma(X, M)\right)
$$

for $v \in S$. Then $\mathrm{H}^{*}\left(U_{v}^{\bullet}\right)=\lim _{n} \mathrm{H}^{*} \Gamma\left(Y_{n}, U_{v}^{\prime \bullet}\right)$ by our dense image assumption and Mittag-Leffler, and it follows that the $\Gamma\left(X, O_{X}\right)$-modules on the left-hand side are coadmissible. The following theorem is again a consequence of the finiteness and pushforward results of Section 1C:

Theorem 1.13. In the situation (4) above, assume the local conditions $\Delta^{\prime}$ are bounded above with transition maps having dense image. Then the natural map

$$
\mathbf{R} \widetilde{\Gamma}_{\mathrm{f}}\left(G_{K, S}, \Gamma(X, M) ; \Delta\right) \rightarrow \mathbf{R} f_{*} \mathbf{R} \widetilde{\Gamma}_{\mathrm{f}}\left(G_{K, S}, M ; \Delta^{\prime}\right)
$$

is an isomorphism in $\mathbf{D}_{\text {coadm }}^{-}(Z)$.

If for each $v \in S$ one has $\left[U_{v}\right] \in \mathbf{D}_{\text {perf }}^{[0,2]}\left(Z^{\prime}\right)$, then the isomorphism takes place in $\mathbf{D}_{\text {perf }}^{[0,3]}(Z)$.

Next we treat arithmetic duality. Recall the anti-involution on perfect complexes $X \mapsto X^{*}=\mathbf{R} \operatorname{Hom}_{O_{Z}}\left(X, O_{Z}\right)=\operatorname{Hom}_{\mathscr{O}_{Z}}\left(X, O_{Z}\right)$. What follows is the basic local result, a variant in families of Tate's local duality theorem. 
Theorem 1.14. (1) For any $v \in S$, there is a canonical isomorphism

$$
\tau_{\geq 2} \mathbf{R} \Gamma_{\text {cont }}\left(G_{v}, \mathrm{O}_{Z}(1)\right) \stackrel{\sim}{\rightarrow} \mathrm{O}_{Z}[-2]
$$

given by base change of the local trace map.

(2) For any $v \in S$, the duality morphism

$$
\mathbf{R} \Gamma_{\text {cont }}\left(G_{v}, M^{*}(1)\right) \rightarrow \mathbf{R} \Gamma_{\text {cont }}\left(G_{v}, M\right)^{*}[-2]
$$

adjoint to the pairing

$$
\begin{aligned}
\mathbf{R} \Gamma_{\text {cont }}\left(G_{v}, M^{*}(1)\right) \otimes_{\mathscr{O}_{Z}}^{\mathbf{L}} \mathbf{R} \Gamma_{\text {cont }}\left(G_{v}, M\right) & \stackrel{\cup}{\rightarrow} \mathbf{R} \Gamma_{\text {cont }}\left(G_{v}, M^{*}(1) \otimes_{\mathcal{O}_{Z}} M\right) \\
& \rightarrow \tau_{\geq 2} \mathbf{R} \Gamma_{\text {cont }}\left(G_{v}, \mathscr{O}_{Z}(1)\right) \stackrel{\sim}{\rightarrow} \mathscr{O}_{Z}[-2],
\end{aligned}
$$

given by cup product, evaluation and truncation, and (1) above, is an isomorphism in $\mathbf{D}_{\text {perf }}^{[0,2]}(Z)$.

Proof. To see (1), we note that, because $G_{v}$ satisfies $e=2$, the Tor-spectral sequence shows that the rule $M \mapsto \mathrm{H}_{\text {cont }}^{2}\left(G_{v}, M\right)$ commutes with arbitrary base change in $Z$. Thus, it suffices to take the composition

$$
\begin{aligned}
\tau_{\geq 2} \mathbf{R} \Gamma_{\text {cont }}\left(G_{v}, \mathrm{O}_{Z}(1)\right) & \cong \mathrm{H}_{\text {cont }}^{2}\left(G_{v}, \mathrm{O}_{Z}(1)\right)[-2] \\
& \cong\left(\mathrm{H}_{\text {cont }}^{2}\left(G_{v}, \mathbf{z}_{p}(1)\right) \otimes_{\mathbf{z}_{p}} \mathrm{O}_{Z}\right)[-2] \\
& \cong\left(\mathbf{Z}_{p} \otimes_{\mathbf{z}_{p}} \mathrm{O}_{Z}\right)[-2]=\mathrm{O}_{Z}[-2],
\end{aligned}
$$

the last identification coming from the trace isomorphism of local class field theory.

To treat (2), we observe that the formation of the duality morphism commutes with arbitrary derived base change in $Z$, and all cases under consideration can be reduced to local scenarios that are pullbacks of the situation (1) on page 1584; hence, it suffices to assume we are in that specific case with $(A, I)$ the ring in question. A morphism of perfect complexes is a quasi-isomorphism if and only if it becomes a quasi-isomorphism after applying $\otimes_{A}^{\mathbf{L}} A / \mathfrak{m}$ for any maximal ideal $\mathfrak{m}$ of $A$. By executing this base change and noting by [Matsumura 1989, Theorem 8.2(i)] that $I \subseteq \mathfrak{m}$, we are reduced to the case where $A$ is a field of characteristic $p$ with the discrete topology. But then $G_{v}$ acts on $M$ via a finite quotient, and the situation arises as the base change of a situation with coefficients in a finite field, where the result is known.

The global result is more complicated to state. As a first approximation, we introduce compactly supported cochains $\mathrm{C}_{\mathrm{cont,c}}^{\bullet}\left(G_{K, S}, M\right)$ as the complex

$$
\text { Cone }\left[\mathrm{C}_{\text {cont }}^{\bullet}\left(G_{K, S}, M\right) \stackrel{\oplus_{v \in S} \text { res }_{v}}{\longrightarrow} \bigoplus_{v \in S} \mathrm{C}_{\text {cont }}^{\bullet}\left(G_{v}, M\right)\right][-1],
$$


denoting its image in the derived category by $\mathbf{R} \Gamma_{\text {cont,c }}\left(G_{K, S}, M\right) \in \mathbf{D}_{\text {perf }}^{[1,3]}(Z)$ and its cohomology by $\mathrm{H}_{\text {cont,c }}^{*}\left(G_{K, S}, M\right) \in \mathbf{K}_{\mathrm{ft}}^{[1,3]}(Z)$. Following Nekovár [2006, 5.3.3.3], one constructs cup product pairings

$$
\mathbf{R} \Gamma_{\text {cont }}\left(G_{K, S}, M\right) \otimes_{\mathscr{O}_{Z}}^{\mathbf{L}} \mathbf{R} \Gamma_{\text {cont, }}\left(G_{K, S}, M^{\prime}\right) \rightarrow \mathbf{R} \Gamma_{\text {cont,c }}\left(G_{K, S}, M \otimes_{\mathscr{O}_{Z}} M^{\prime}\right),
$$

and the following theorem is proved in the exact same way as the preceding one:

Theorem 1.15. (1) There is a canonical isomorphism

$$
\tau_{\geq 3} \mathbf{R} \Gamma_{\text {cont, }}\left(G_{K, S}, \mathrm{O}_{Z}(1)\right) \stackrel{\sim}{\rightarrow} \mathrm{O}_{Z}[-3]
$$

given by base change of the global trace map.

(2) For any $v \in S$, the duality morphism

$$
\mathbf{R} \Gamma_{\text {cont }}\left(G_{K, S}, M^{*}(1)\right) \rightarrow \mathbf{R} \Gamma_{\text {cont, }}\left(G_{K, S}, M\right)^{*}[-3]
$$

adjoint to the pairing

$$
\begin{aligned}
\mathbf{R} \Gamma_{\text {cont }}\left(G_{K, S}, M^{*}(1)\right) \otimes_{\mathscr{O}_{Z}}^{\mathbf{L}} \mathbf{R} \Gamma_{\text {cont,c }}\left(G_{K, S}, M\right) \\
\stackrel{\cup}{\rightarrow} \mathbf{R} \Gamma_{\text {cont,c }}\left(G_{K, S}, M^{*}(1) \otimes_{\mathcal{O}_{Z}} M\right) \\
\rightarrow \tau_{\geq 3} \mathbf{R} \Gamma_{\text {cont, }}\left(G_{K, S}, \mathcal{O}_{Z}(1)\right) \stackrel{\sim}{\rightarrow} \mathscr{O}_{Z}[-3],
\end{aligned}
$$

given by cup product, evaluation and truncation, and (1) above, is an isomorphism in $\mathbf{D}_{\text {perf }}^{[0,2]}(Z)$.

To treat duality of Selmer complexes, assume the local conditions $\Delta$ satisfy $\left[U_{v}\right] \in \mathbf{D}_{\text {perf }}^{[0,2]}(Z)$ for all $v \in S$, and equip $M^{*}(1)$ with local conditions $\Delta^{*}(1)$ given for $v \in S$ by choosing a representative in $\mathbf{K}_{\text {perf }}^{[0,2]}(Z)$ of the morphism

$$
E_{v}^{*}[-2] \stackrel{j_{v}^{*}[-2]}{\longrightarrow} \mathbf{R} \Gamma_{\text {cont }}\left(G_{v}, M\right)^{*}[-2] \cong \mathbf{R} \Gamma_{\text {cont }}\left(G_{v}, M^{*}(1)\right) .
$$

Then as in [Nekováŕ 2006, 6.3], one constructs cup product pairings

$\mathbf{R} \widetilde{\Gamma}_{\mathrm{f}}\left(G_{K, S}, M^{*}(1) ; \Delta^{*}(1)\right) \otimes_{\mathscr{O}_{Z}}^{\mathbf{L}} \mathbf{R} \widetilde{\Gamma}_{\mathrm{f}}\left(G_{K, S}, M ; \Delta\right)$

$$
\rightarrow \mathbf{R} \Gamma_{\text {cont, }}\left(G_{K, S}, M^{*}(1) \otimes_{\mathbb{O}_{Z}} M\right),
$$

which, followed by evaluation and truncation, followed by the global trace map, gives rise via adjoint to a duality morphism

$$
\mathbf{R} \widetilde{\Gamma}_{\mathrm{f}}\left(G_{K, S}, M^{*}(1) ; \Delta^{*}(1)\right) \rightarrow \mathbf{R} \widetilde{\Gamma}_{\mathrm{f}}\left(G_{K, S}, M ; \Delta\right)^{*}[-3] .
$$

The general global result is as follows, with the same proof.

Theorem 1.16. Assume, for all $v \in S$, the local conditions satisfy $\left[U_{v}\right] \in \mathbf{D}_{\text {perf }}^{[0,2]}(Z)$. Then the duality morphism above is an isomorphism in $\mathbf{D}_{\text {perf }}^{[0,3]}(Z)$. 
Remark 1.17. The preceding base-changing and duality theorems are cheating because we have taken a narrow definition of "local condition". Rather than a particular morphism $i_{v}$, the phrase usually means a rule that associates such a morphism to a given $M$ (perhaps equipped with additional data) as in the examples below. The true base-changing and duality theorems are reduced by the above theorems to the claim that the formation of the local conditions, as determined by the rule, commutes with base change and duality, respectively.

Example 1.18. We give some examples of useful local conditions. Let $v \in S$.

The empty local condition means taking $i_{v}$ to be the identity map and results in no modification being made to the cohomology. The full local condition, taking $i_{v}$ to be the map from the zero object, results in cohomology that is "compactly supported" at $v$. The formation of these conditions clearly commutes with arbitrary base change. Excepting these two, a local condition often has the property that $\mathrm{H}^{0}\left(i_{v}\right)$ is an isomorphism, $\mathrm{H}^{1}\left(i_{v}\right)$ is an injection, and $\mathrm{H}^{n}\left(U_{v}\right)=0$ for $n \neq 0,1$. In fact, given a subspace $\mathscr{L} \subseteq \mathrm{H}_{\text {cont }}^{1}\left(G_{v}, M\right)$, there is a standard construction of such a local condition with img $\mathrm{H}^{1}\left(i_{v}\right)=\mathscr{L}$, called the local condition associated to $\mathscr{L}$, namely by setting $U_{v}^{0}=\mathrm{C}_{\text {cont }}^{0}\left(G_{v}, M\right)$,

$$
U_{v}^{1}=\left\{c \in \mathrm{C}_{\mathrm{cont}}^{1}\left(G_{v}, M\right) \mid d c=0,[c] \in \mathscr{L}\right\},
$$

and $U_{v}^{n}=0$ for $n \neq 0,1$ (and taking $i_{v}$ to be the inclusion map of complexes). On the other hand, when $\mathrm{H}^{1}\left(i_{v}\right)$ fails to be injective, its kernel is considered a local contribution to an "exceptional zero" of the related $p$-adic L-function.

For $v \in S$ not dividing $p$, the unramified local condition is given by inflation

$$
i_{v}: U_{v}^{\bullet}=\mathrm{C}_{\text {cont }}^{\bullet}\left(G_{v} / I_{v}, M^{I_{v}}\right) \rightarrow \mathrm{C}_{\text {cont }}^{\bullet}\left(G_{v}, M\right) .
$$

(Note that $G_{v} / I_{v} \approx \widehat{\mathbf{Z}}$ satisfies Hypothesis (1) with $e=1$.) It is isomorphic in the derived category to the local condition associated to the image of inflation $\mathrm{H}_{\text {cont }}^{1}\left(G_{v} / I_{v}, M^{I_{v}}\right) \hookrightarrow \mathrm{H}_{\text {cont }}^{1}\left(G_{v}, M\right)$. Whenever $M^{I_{v}}$ is flat over $\mathrm{O}_{Z}$, it obeys the necessary hypotheses as a continuous $\mathrm{O}_{Z}\left[G_{v} / I_{v}\right]$-module. If $f^{*}\left(M^{I_{v}}\right) \stackrel{\sim}{\rightarrow}\left(f^{*} M\right)^{I_{v}}$, then the formation of the unramified local condition commutes with (derived) base change. When both $M^{I_{v}}$ and $M^{*}(1)^{I_{v}}$ are flat over $\mathrm{O}_{Z}$, it makes sense to ask whether the unramified local conditions for $M$ and $M^{*}(1)$ are self-dual, and this seems to be the case only generically: see, for example, [Nekováŕ 2006, 7.6 and 7.6.7(iii)] for closely related statements in the situation (1) on page 1584 with $I$ a maximal ideal (where our *-operation has been replaced by Grothendieck duality, which is not a change when $A$ is Gorenstein).

We warn the reader that in general $M^{I_{v}}$ need not be flat, in which case its finiteness and base-changing properties become much more subtle. There is no problem for families of twists of a fixed global Galois representation; for example, 
in Example 1.19(1) below, one has $M^{I_{v}}=T^{I_{v}} \otimes \mathbf{z}_{p} A$. But the complication does arise in Hida theory, say in Example 1.19(2) below, when a $p$-ordinary eigenform admits level lowering modulo $p$ at a prime $v=\ell \neq p$. Then there are $\ell$-old and $\ell$-new branches of the Hida family, over which $M^{I_{v}}$ has generic rank, respectively, two and strictly less than two. Since geometrically these branches meet in the special fiber of Spec $\mathfrak{h}_{\infty}^{\text {ord }}$, the module $M^{I_{v}}$ cannot be locally free. This phenomenon is related to the appearance of $p$ in the Tamagawa number at $\ell$ because the latter occurrence can be used to detect level-lowering.

For $v$ dividing $p$, most local conditions are rather complicated; even the determination of a meaningful subspace $\mathscr{L}$ to which to associate them is rather delicate. The best-behaved notion is the (strict) ordinary one: one assumes given a $G_{v^{-}}$stable locally direct summand $M_{v}^{+} \subseteq M$ and takes $\Delta_{v}$ to be the data of the natural map

$$
i_{v}: U_{v}^{\bullet}=\mathrm{C}_{\text {cont }}^{\bullet}\left(G_{v}, M_{v}^{+}\right) \rightarrow \mathrm{C}_{\text {cont }}^{\bullet}\left(G_{v}, M\right) .
$$

This local condition appears in work of Nekovár [2006, 6.7] as a derived variant of the subspace $\mathscr{L}$ given by the image of $\mathrm{H}_{\text {cont }}^{1}\left(G_{v}, M_{v}^{+}\right) \rightarrow \mathrm{H}_{\text {cont }}^{1}\left(G_{v}, M\right)$ used by Greenberg [1989; 1994a; 1994b]; they are frequently isomorphic in the derived category for example as in Proposition 3.7(3) below. The formation of the ordinary local condition commutes with base change and is dual to the ordinary local condition formed with the annihilator of $M_{v}^{+}$in $M^{*}(1)$. The abstract base change and duality theorems recover Greenberg's control and duality theorems in the situation (1), with $A$ local with finite residue field, and gives analogues of it in all cases. All this presupposes the existence of a useful choice of $M_{v}^{+}$to begin with; the key observation of this article is that, after replacing the $G_{v}$-module $M$ by its associated $\left(\varphi, \Gamma_{K_{v}}\right)$-module, one can still form an ordinary local condition, and one gains access to subobjects of $\mathbf{D}_{\mathrm{pst}}(M)$ that are not necessarily weakly admissible.

Example 1.19. Take $K=\mathbf{Q}$ for simplicity. The present results, notably situation (5), apply to the following settings. Each $A$ is local, we take $I$ to be its maximal ideal, and $A / I$ is a finite field. We write $X$ for the generic fiber of $\mathfrak{X}=\operatorname{Spf}(A, I)$.

(1) $A$ is the Iwasawa algebra $\mathbf{Z}_{p} \llbracket \Gamma \rrbracket$, where $\Gamma=\operatorname{Gal}\left(\mathbf{Q}_{\infty} / \mathbf{Q}\right)$ is the Galois group of the cyclotomic $\mathbf{Z}_{p}$-extension $\mathbf{Q}_{\infty} / \mathbf{Q}$, and $M=T \otimes_{\mathbf{z}_{p}} A$ with diagonal $G_{\mathbf{Q}, S}$-action, where $T$ is a continuous $\mathbf{Z}_{p}\left[G_{\mathbf{Q}, S}\right]$-module that is free over $\mathbf{Z}_{p}$. Thus, $M$ is the cyclotomic deformation of $T$, and the parameter space $X$ is commonly called the weight space.

(2) $A$ is a Hida-Hecke algebra $\mathfrak{h}_{\infty}^{\text {ord }}$, and $M$ is Hida's $\Lambda$-adic Galois representation (with $A$ assumed Gorenstein so that $M$ is flat). Thus, $X$ is commonly called the ordinary locus of the Coleman-Mazur eigencurve. 
(3) $A$ is any $\mathbf{Z}_{p}$-flat quotient of a Galois deformation ring $R^{\text {univ }}(\bar{\rho})$, where $\bar{\rho}$ is an absolutely irreducible mod $p$ representation of $G_{\mathbf{Q}, S}$, and $M$ is deduced from the universal deformation of $\bar{\rho}$.

Traditionally, the algebraic part of Iwasawa theory concerns the study of Selmer groups over $A$ in the above examples. Theorem 1.9 translates this study to $X$, with the loss of only $p$-torsion information, as $\Gamma\left(X, \mathscr{O}_{X}\right)$ is faithfully flat over $A[1 / p]$. Since $(\varphi, \Gamma)$-modules over the Robba ring (which we use to generalize Greenberg's "ordinary" theory) form families over $X$ and not over $\mathfrak{X}$, this change of view makes it now possible to treat nonordinary situations.

\section{2. $(\varphi, \Gamma)$-modules and Galois cohomology}

In this section, we fix a finite extension $K$ of $\mathbf{Q}_{p}$ with ring of integers $\mathbb{O}_{K}$ and residue field $k$. We choose an algebraic closure $K^{\text {alg }}$ of $K$ and set $G=G_{K}=\operatorname{Gal}\left(K^{\text {alg }} / K\right)$. (Several of the techniques discussed below will be valid if $K$ is replaced by a general complete, discretely valued field of mixed characteristic $(0, p)$ and perfect residue field, but beware that the group $G$ satisfies Hypothesis (1) only when $K$ is finite over $\mathbf{Q}_{p}$, which gets in the way of certain base-change arguments.)

We let $A^{\prime}$ be a Noetherian commutative $E$-Banach algebra, having $A$ as its unit ball, so that $A^{\prime}=A[1 / p]$ and $A$ satisfies Hypothesis (2) when equipped with the ideal $I=(p)$. Finally, we let $M$ satisfy Hypotheses A(3)-(4).

2A. Recall of $\varphi$ - and $(\varphi, \Gamma)$-modules. There are several variants of $\left(\varphi, \Gamma_{K}\right)$-modules, so we must recall several base rings. We only do this minimally since they are defined in many places now (see, e.g., [Berger 2002]). For any field $F$, write $F_{n}=F\left(\mu_{p^{n}}\right)$ for $n \leq \infty$.

Let $F=$ Frac W $(k)$. If $k^{\prime}$ denotes the residue field of $K_{\infty}$, define $F^{\prime}=\operatorname{Frac} \mathrm{W}\left(k^{\prime}\right)$ and $K^{\prime}=K . F^{\prime}$. We set $H=H_{K}=\operatorname{Gal}\left(K^{\text {alg }} / K_{\infty}\right)$ and $\Gamma=\Gamma_{K}=\operatorname{Gal}\left(K_{\infty} / K\right)$. The group $\Gamma$ is either procyclic or of the form $\{ \pm 1\} \times$ (procyclic); we let $\Delta=\Delta_{K} \subset \Gamma$ be trivial in the first case and $\{ \pm 1\}$ in the second case.

There is an increasing system of $p$-torsion-free, $p$-adically separated, and complete $\mathrm{W}\left(k^{\text {alg }}\right)$-algebras $\widetilde{\mathrm{A}}^{\dagger, s}$ equipped with a compatible action of $G$, indexed by real numbers $s>0$. This family is also equipped with an automorphism $\varphi$ that commutes with $G$ and takes $\widetilde{\mathrm{A}}^{\dagger, s}$ onto $\widetilde{\mathrm{A}}^{\dagger, p s}$. Each of the $p$-adic Banach algebras $\widetilde{\mathrm{B}}^{\dagger, s}=\widetilde{\mathrm{A}}^{\dagger, s}[1 / p]$ admits a certain Fréchet completion $\widetilde{\mathrm{B}}_{\text {rig }}^{\dagger, s}$, to which both actions extend uniquely by continuity, and these latter rings also fit into an increasing system. One defines the systems $\widetilde{\mathrm{A}}_{K}^{\dagger, s}=\left(\widetilde{\mathrm{A}}^{\dagger, s}\right)^{H}, \widetilde{\mathrm{B}}_{K}^{\dagger, s}=\left(\widetilde{\mathrm{B}}^{\dagger, s}\right)^{H}$, and $\widetilde{\mathrm{B}}_{\text {rig }, K}^{\dagger, s}=\left(\widetilde{\mathrm{B}}_{\text {rig }}^{\dagger, s}\right)^{H}$ with the induced topologies and actions of $\varphi$ and $\Gamma$.

The theory of the field of norms allows one to make a choice of a sort of indeterminate $\pi_{K}$ belonging to all the $\tilde{\mathrm{A}}_{K}^{\dagger, s}$ and associates to $K$ a constant $\mathrm{e}_{K}>0$. When $K=F$, there is an almost canonical choice that is written $\pi$, and one can 
calculate that $\mathrm{e}_{F}=p /(p-1)$. For $s>0$, one has subrings

$$
\begin{gathered}
\mathrm{B}_{K}^{\dagger, s}=\left\{f\left(\pi_{K}\right)=\sum_{n \in \mathbf{Z}} a_{n} \pi_{K}^{n} \mid \begin{array}{c}
a_{n} \in F^{\prime},\left\{\left|a_{n}\right|\right\} \text { bounded, } \\
f(X) \text { convergent for } 0<\operatorname{ord}_{p}(X)<1 / \mathrm{e}_{K} s
\end{array}\right\}, \\
\mathrm{B}_{\text {rig, }, K}^{\dagger, s}=\left\{\begin{array}{c|c}
a_{n} \in F^{\prime}, \\
f\left(\pi_{K}\right)=\sum_{n \in \mathbf{Z}} a_{n} \pi_{K}^{n} & f(X) \text { convergent for } 0<\operatorname{ord}_{p}(X)<1 / \mathrm{e}_{K} s
\end{array}\right\}
\end{gathered}
$$

of $\widetilde{\mathrm{B}}_{K}^{\dagger, s}$ and $\widetilde{\mathrm{B}}_{\mathrm{rig}, K}^{\dagger, s}$, respectively, that do not depend on the choice of $\pi_{K}$ for $s \gg 0$. They inherit topologies, and for $s \gg 0$, they are stable under $\Gamma$, and $\varphi$ sends $\mathrm{B}_{K}^{\dagger, s}$ into $\mathrm{B}_{K}^{\dagger, p s}$ and $\mathrm{B}_{\text {rig, } K}^{\dagger, s}$ into $\mathrm{B}_{\mathrm{rig}, K}^{\dagger, p s}$. One knows that $\varphi$ acts by Witt functoriality on $a_{n} \in F^{\prime}$, and $\Gamma$ acts on $a_{n}$ through its quotient $\Gamma_{K} / \Gamma_{K^{\prime}}=\operatorname{Gal}\left(F^{\prime} / F\right)$. The action on $\pi_{K}$ is generally not explicitly given (especially since there is some choice in $\left.\pi_{K}\right)$ except when $K=F$, in which case $\varphi(\pi)=(1+\pi)^{p}-1$ and $\gamma \in \Gamma$ obeys $\gamma(\pi)=(1+\pi)^{x_{\text {cycl }}(\gamma)}-1$. In any case, $\varphi$ now induces a finite free algebra extension of degree $p$ for $s \gg 0$ instead of being an isomorphism. For such $s$, we obtain a left inverse $\psi: \mathrm{B}_{(\text {rig, }) K}^{\dagger, p s} \rightarrow \mathrm{B}_{(\text {rig, }) K}^{\dagger, s}$ to $\varphi$ by the formula $p^{-1} \varphi^{-1} \circ \operatorname{Tr}_{\mathrm{B}_{(\mathrm{rig},) K}^{\dagger, p s} / \varphi \mathrm{B}_{(\mathrm{rig},) K}^{\dagger \dagger s}}^{\mathrm{B}^{\dagger}}$.

For any of the above families of rings, we denote the result of applying $\lim _{s}$ by omitting the index $s$, e.g., $\mathrm{B}_{\mathrm{rig}, K}^{\dagger}=\lim _{\rightarrow} \mathrm{B}_{\mathrm{rig}, K}^{\dagger, s}$. The result inherits the direct limit topology, an action of $G$, a ring endomorphism $\varphi$, and a left inverse $\psi$. The ring $\mathrm{B}_{K}^{\dagger}$ is the fraction field of a Henselian, mixed-characteristic discrete valuation ring with imperfect residue field (although we never make use of the topology it would provide). Although the rings $\mathrm{B}_{\mathrm{rig}, K}^{\dagger(, s)}$ are non-Noetherian, they are Bézout domains. For brevity, we will often denote an unspecified one of the rings $A^{\prime} \widehat{\otimes}_{\mathbf{Q}_{p}} \mathrm{~B}_{K}^{\dagger(, s)}$, $A^{\prime} \widehat{\otimes}_{\mathbf{Q}_{p}} \widetilde{\mathrm{B}}_{K}^{\dagger(, s)}$, or $A^{\prime} \widehat{\otimes}_{\mathbf{Q}_{p}} \mathrm{~B}_{\text {rig, }, K}^{\dagger(, s)}$ simply by $\mathbf{B}^{(s)}$, and when we must emphasize its dependence on $K$, we will write $\mathrm{B}_{K}^{(s)}$.

If $L / K$ is a finite Galois extension inside $K^{\text {alg }}$, then one can arrange for $\pi_{L}$ to satisfy an Eisenstein polynomial over a subring of $\mathrm{B}_{K}^{\dagger} \otimes_{F^{\prime}} F_{L}^{\prime}$ with respect to a suitable $\pi_{K}$-adic valuation. (The term $F_{L}^{\prime}$ is the maximal absolutely unramified subfield of $L_{\infty}$ analogous to $F^{\prime}$.) The constants $\mathrm{e}_{K}$ and $\mathrm{e}_{L}$ are normalized so that the growth conditions on power series coincide. For $s \gg 0$, one gets functorial embeddings $\mathrm{B}_{K}^{(s)} \hookrightarrow \mathrm{B}_{L}^{(s)}$, which are finite free ring extensions (for $s \gg 0$ ) compatible with the actions of $\varphi$ and $\Gamma_{L}$, and thus an action of $H_{K} / H_{L}$ on $\mathrm{B}_{L}^{(s)}$ with $\left(\mathrm{B}_{L}^{(s)}\right)^{H_{K}}=\mathrm{B}_{K}^{(s)}$.

The series $\log (1+\pi)=\sum_{n \geq 1} \frac{(-1)^{n-1}}{n} \pi^{n}$ converges in $\mathrm{B}_{\text {rig, } F}^{\dagger, s}$ for every $s>0$, and we call its limit $t$. By means of the above embedding process, $t$ is an element of every $\mathrm{B}_{\mathrm{rig}, K}^{\dagger, s}$. One has $\varphi(t)=p t$ and $\gamma(t)=\chi_{\mathrm{cycl}}(\gamma) t$ for all $\gamma \in \Gamma$.

Given a $\mathrm{B}^{s}$-module $D^{s}$, write $D^{\left(s^{\prime}\right)}=D \otimes_{\mathrm{B}^{s}} \mathrm{~B}^{\left(s^{\prime}\right)}$ for $s^{\prime} \geq s$. A $\varphi$-module over $\mathrm{B}^{(s)}$ is a finitely presented, projective $\mathrm{B}^{(s)}$-module $D^{(s)}$ equipped with a semilinear map $\varphi: D^{(s)} \rightarrow D^{(p s)}$ such that the associated linear map $\varphi^{\prime}: \mathrm{B}^{(p s)}{ }_{\varphi} \otimes_{\mathrm{B}^{(s)}} D^{(s)} \rightarrow D^{(p s)}$ is an isomorphism. We write $\mathbf{M}(\varphi) / \mathrm{B}^{(s)}$ or $\mathbf{M}(\varphi)$ for the exact category of $\varphi$-modules over $\mathrm{B}^{(s)}$. A $(\varphi, \Gamma)$-module over $\mathrm{B}^{(s)}$ is a $\varphi$-module $D^{(s)}$ over $\mathrm{B}^{(s)}$ equipped with 
a semilinear action of $\Gamma$ that commutes with $\varphi$ and is continuous for varying $\gamma \in \Gamma$. We write $\mathbf{M}(\varphi, \Gamma) \mathrm{B}^{(s)}$ or $\mathbf{M}(\varphi, \Gamma)$ for the exact category of $(\varphi, \Gamma)$-modules over $\mathrm{B}^{(s)}$; it has tensor products and internal homs. Ultimately, we are concerned with $(\varphi, \Gamma)$-modules over $A^{\prime} \widehat{\otimes}_{\mathbf{Q}_{p}} \mathrm{~B}_{\mathrm{rig}, K}^{\dagger}$ because it is over this ring that the link to $p$-adic Hodge theory is direct, and a finiteness theorem is known for Galois cohomology in complete generality. But for technical reasons, we must make use of the other variants at times; especially, the construction of the functor from Galois representations to $(\varphi, \Gamma)$-modules is documented in the literature in terms of $A^{\prime} \widehat{\otimes}_{\mathbf{Q}_{p}} \mathbf{B}_{K}^{\dagger}$, and our proof of this functor's compatibility of Galois cohomology makes use of $A^{\prime} \widehat{\otimes} \mathbf{Q}_{p} \widetilde{\mathrm{B}}_{K}^{\dagger, s}$.

Suppose that $s \gg 0$ so that $\psi: \mathrm{B}_{(\text {rig, }) K}^{\dagger, p s} \rightarrow \mathrm{B}_{(\text {rig, }) K}^{\dagger, s}$ is defined and hence also a left inverse $1 \widehat{\otimes} \psi: \mathrm{B}^{p s} \rightarrow \mathrm{B}^{s}$ to $1 \widehat{\otimes} \varphi: \mathrm{B}^{s} \rightarrow \mathrm{B}^{p s}$, and let $D^{s}$ be a $\varphi$-module over $A^{\prime} \widehat{\otimes}_{\mathbf{Q}_{p}} \mathrm{~B}_{(\text {rig, }) \mathbf{Q}_{p}}^{\dagger, s}$. Then we obtain a left inverse to $\varphi$ on $D^{s}$ by the rule

$$
D^{p s} \widetilde{\mathrm{B}^{p s}} \otimes_{\varphi, \mathrm{B}^{s}} D^{s} \stackrel{(1 \widehat{\otimes} \psi) \otimes 1}{\longrightarrow} \mathrm{B}^{s} \otimes_{\mathrm{B}^{s}} D^{s}=D^{s} .
$$

Upon taking $\lim _{\rightarrow}$, one gets a map $\psi: D \rightarrow D$ that is also left inverse to $\varphi$.

We will make use of the slope theory for $\varphi$-modules as in the following:

Theorem 2.1 [Kedlaya 2008]. There is a homomorphism deg : $\left(\mathrm{B}_{\mathrm{rig}, K}^{\dagger}\right)^{\times} \rightarrow \mathbf{Q}$ extending $\operatorname{ord}_{p}$ with the property that the rule $\operatorname{deg}(D)=\operatorname{deg}(\varphi \mid \operatorname{det} D)$ gives rise to a theory of Harder-Narasimhan filtrations on $\mathbf{M}(\varphi) / \mathrm{B}_{\mathrm{rig}, K}^{\dagger}$.

One calls a $\varphi$-module over $\mathrm{B}_{\mathrm{rig}, K}^{\dagger}$ étale if its only slope is 0 . The full subcategory of étale $\varphi$-modules is denoted by $\mathbf{M}^{\text {ét }}(\varphi) \subset \mathbf{M}(\varphi)$. A $(\varphi, \Gamma)$-module $D$ is called étale if its underlying $\varphi$-module is; the full subcategory of these is written $\mathbf{M}^{\mathrm{e}} \mathrm{t}(\varphi, \Gamma) \subset \mathbf{M}(\varphi, \Gamma)$. Since the slope filtration is unique, it is $\Gamma$-stable.

We write $\operatorname{Rep}_{A}(G)$ and $\operatorname{Rep}_{A^{\prime}}(G)$ for the category of finitely generated, flat $A$-modules and $A^{\prime}$-modules, respectively, equipped with a continuous, linear action of $G$.

Theorem 2.2. Let $M \in \operatorname{Rep}_{A^{\prime}}(G)$. For $s \gg 0$, there exists a canonical $\varphi$-and G-stable $A \widehat{\otimes} \mathbf{z}_{p} \mathrm{~B}_{K}^{\dagger, s}$-submodule

$$
\mathbf{D}^{\dagger, s}(M) \subseteq\left(M \otimes_{A^{\prime}}\left(A^{\prime} \widehat{\otimes}_{\mathbf{Q}_{p}} \widetilde{\mathrm{B}}^{\dagger, s}\right)\right)^{H}
$$

that is projective of the same rank as $M$ such that the natural map

$$
\left(A^{\prime} \widehat{\otimes}_{\mathbf{Q}_{p}} \widetilde{\mathrm{B}}^{\dagger, s}\right) \otimes_{\left(A^{\prime} \widehat{\otimes}_{\mathbf{Q}_{p}} \mathrm{~B}_{K}^{\dagger, s}\right)} \mathbf{D}^{\dagger, s}(M) \rightarrow M \otimes_{A^{\prime}}\left(A^{\prime} \widehat{\otimes}_{\mathbf{Q}_{p}} \widetilde{\mathrm{B}}^{\dagger, s}\right)
$$

is an isomorphism; $\mathbf{D}^{\dagger, s}(M)$ is a $(\varphi, \Gamma)$-module over $A^{\prime} \widehat{\otimes}_{\mathbf{Q}_{p}} \mathbf{B}_{K}^{\dagger, s}$. (By “ $\varphi$-stable", we mean that $\varphi \mathbf{D}^{\dagger, s}(M) \subseteq\left(A^{\prime} \widehat{\otimes}_{\mathbf{Q}_{p}} \mathbf{B}_{K}^{\dagger, p s}\right) \cdot \mathbf{D}^{\dagger, s}(M)$.) If $M$ is of the form $M_{0}[1 / p]$ where $M_{0} \in \operatorname{Rep}_{A}(G)$ is free and such that $G$ acts trivially on $M_{0} / 12 p$, then 
$\mathbf{D}^{\dagger, s}(M)$ is in fact free over $\mathbf{B}_{K}^{\dagger, s}$. If $B$ is a $p$-adic A-algebra and $B^{\prime}=B[1 / p]$, then the natural maps

$$
\mathbf{D}^{(s)}(M) \widehat{\otimes}_{A^{\prime}} B^{\prime} \rightarrow \mathbf{D}^{(s)}\left(M \otimes_{A^{\prime}} B^{\prime}\right)
$$

are isomorphisms, provided $s \gg 0$ so that both sides exist.

Proof. Except for the final claim of compatibility with base change along $A^{\prime} \rightarrow B^{\prime}$, this follows from [Berger and Colmez 2008, Proposition 4.2.8 and Théorème 4.2.9] when $M$ is free and from [Kedlaya and Liu 2010, Theorem 3.11 and Definition 3.12] in general. The final claim follows directly from the constructions although this is never explicitly stated in either reference.

For $M$ as in the theorem, we define

$$
\begin{aligned}
& \widetilde{\mathbf{D}}^{\dagger(, s)}(M)=\mathbf{D}^{\dagger, s}(M) \otimes_{\left(A \widehat{\otimes}_{\mathbf{z}_{p}} \mathrm{~B}_{K}^{\dagger, s}\right)}\left(A \widehat{\otimes} \mathbf{z}_{p} \widetilde{\mathrm{B}}_{K}^{\dagger(, s)}\right), \\
& \mathbf{D}_{\text {rig }}^{\dagger(, s)}(M)=\mathbf{D}^{\dagger, s}(M) \otimes_{\left(A \widehat{\otimes} \mathbf{z}_{p} \mathrm{~B}_{K}^{\dagger, s}\right)}\left(A \widehat{\otimes} \mathbf{z}_{p} \mathrm{~B}_{\mathrm{rig}, K}^{\dagger(, s)}\right) .
\end{aligned}
$$

For brevity, we will often denote the above associated module corresponding to the ring $\mathrm{B}^{(s)}$ by $\mathbf{D}^{(s)}(M)$ and a general $\mathrm{B}^{(s)}$-module by $D^{(s)}$.

Theorem 2.3. For each ring $\mathrm{B}^{(s)}$, the rule $M \mapsto \mathbf{D}(M)$ determines an exact functor $\operatorname{Rep}_{A^{\prime}}(G) \rightarrow \mathbf{M}(\varphi, \Gamma) / \mathrm{B}$ respecting tensor and internal hom structures. Assuming additionally that $A^{\prime}$ is an affinoid algebra, this functor is fully faithful. When $A$ is finite over $\mathrm{O}_{E}$, the essential image of $\mathbf{D}_{\text {rig }}^{\dagger}$ is $\mathbf{M}^{\mathrm{e} t}(\varphi, \Gamma)_{/ \mathrm{B}}$.

Proof. The full faithfulness of $\mathbf{D}^{\dagger}$ is given by [Kedlaya and Liu 2010, Proposition 2.7] and the comment of [ibid., Definition 3.12], and the full faithfulness of $\widetilde{\mathbf{D}}^{\dagger}$ follows by the same argument. The full faithfulness of $\mathbf{D}_{\text {rig }}^{\dagger}$ is given by [ibid., Proposition 6.5]. The remaining claims are straightforward.

Let $L / K$ be a finite Galois extension inside $K^{\text {alg }}$. For a $\mathrm{B}_{K}^{(s)}$-module $D^{(s)}$, we use the shorthand $D_{L}^{(s)}=D^{(s)} \otimes_{\mathrm{B}_{K}^{(s)}} \mathrm{B}_{L}^{(s)}$. If $D^{(s)}$ has a $\varphi$-action, so does $D_{L}^{(s)}$. If $D^{(s)}$ has a $\Gamma_{K}$-action, then $D_{L}^{(s)}$ has a $\Gamma_{L}$-action. For $M \in \operatorname{Rep}_{A^{\prime}}\left(G_{K}\right)$, one has $\mathbf{D}^{(s)}\left(\left.M\right|_{G_{L}}\right)=\mathbf{D}^{(s)}(M)_{L}$.

The above results suggest the following (ad hoc) formalism. Let $X$ be a $p$-adic analytic space over $E$, and let $U$ be an admissible affinoid covering that is quasiclosed under intersections. For each choice of ring $\mathrm{B}^{(s)}=\mathrm{B}_{K}^{\dagger(s)}, \widetilde{\mathrm{B}}_{K}^{\dagger(s)}, \mathrm{B}_{\text {rig, } K}^{\dagger(s)}$, denote by $\widehat{O}_{u} \widehat{\otimes}_{\mathbf{Q}_{p}} \mathbf{B}^{(s)}$ the sheaf of rings on $\mathcal{U}$ (as always with the discrete Grothendieck topology) determined by the rule

$$
\Gamma\left(Y, \mathrm{O}_{\imath} \widehat{\otimes}_{\mathbf{Q}_{p}} \mathrm{~B}^{(s)}\right)=\Gamma\left(Y, \widehat{O}_{Y}\right) \widehat{\otimes}_{\mathbf{Q}_{p}} \mathbf{B}^{(s)},
$$

equipped with the obvious actions of $\varphi$ and $\Gamma$. If $D^{s}$ is a $\left(\mathcal{O}_{\imath} \widehat{\otimes}_{\mathbf{Q}_{p}} \mathrm{~B}^{s}\right)$-module, then $D^{\left(s^{\prime}\right)}$, interpreted in the obvious manner, is naturally an $\mathcal{O}_{q} \widehat{\otimes}_{\mathbf{Q}_{p}} \mathrm{~B}^{\left(s^{\prime}\right)}$-module for any $s^{\prime}>s$. However, we warn that an $\mathrm{O}_{q} \widehat{\otimes}_{\mathbf{Q}_{p}}$ B-module need not conversely arise 
from any $\mathrm{O}_{u} \widehat{\otimes}_{\mathbf{Q}_{p}} \mathrm{~B}^{s}$-module because the necessary $s$ might not be bounded above for varying $Y \in \mathcal{U}$. Similarly, the natural injection

$$
\underset{s}{\lim } \Gamma\left(\mathcal{U}, \mathscr{O}_{u} \widehat{\otimes}_{\mathbf{Q}_{p}} \mathrm{~B}^{s}\right) \rightarrow \Gamma\left(\mathcal{U}, \mathrm{O}_{u} \widehat{\otimes}_{\mathbf{Q}_{p}} \mathrm{~B}\right)
$$

need not be a bijection. By a family of $\varphi$-modules over $X$ (of type $\mathrm{B}^{(s)}$ ), we mean a quasiconsistent sheaf $D^{(s)}$ of finitely presented flat $\left(\mathcal{O}_{\boldsymbol{U}} \widehat{\otimes}_{\mathbf{Q}_{p}} \mathrm{~B}^{(s)}\right)$-modules equipped with a semilinear action of $\varphi$ such that for each $Y \in \mathcal{U}$ the associated linear map

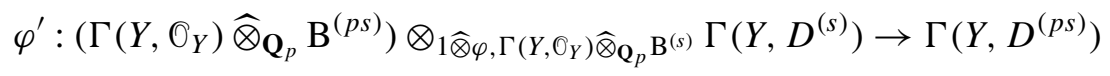

is an isomorphism. By a family of $(\varphi, \Gamma)$-modules over $X$ (of type $\mathrm{B}^{(s)}$ ), we mean a family of $\varphi$-modules $D^{(s)}$ over $X$ (of type $\mathrm{B}^{(s)}$ ) equipped with a semilinear action of $\Gamma$ that commutes with $\varphi$ and is continuous for varying $\gamma \in \Gamma$. Given a family $M$ of $G$-representations over $X$, the rule that associates to $Y \in \mathcal{U}$ the $(\varphi, \Gamma)$-module $\mathbf{D}(\Gamma(Y, M))$ determines a family $\mathbf{D}(M)$ of $(\varphi, \Gamma)$-modules over $X$.

2B. Galois cohomology of $(\varphi, \Gamma)$-modules. For $D^{(s)}$ a $(\varphi, \Gamma)$-module over $\mathrm{B}^{(s)}$, we define its Herr complex [Herr 1998] or Galois cochain complex to be the object

$$
\begin{aligned}
\mathbf{R} \Gamma\left(G, D^{(s)}\right) & =\mathbf{R} \Gamma_{\text {cont }}\left(\Gamma, \operatorname{Cone}\left[D^{(s)} \stackrel{\varphi-1}{\longrightarrow} D^{(p s)}\right][-1]\right) \\
& \cong \operatorname{Cone}\left[\mathbf{R} \Gamma_{\text {cont }}\left(\Gamma, D^{(s)}\right) \stackrel{\varphi-1}{\longrightarrow} \mathbf{R} \Gamma_{\text {cont }}\left(\Gamma, D^{(p s)}\right)\right][-1]
\end{aligned}
$$

of $\mathbf{D}^{\mathrm{b}}\left(A^{\prime}\right)$ and its Galois cohomology $\mathrm{H}^{*}\left(G, D^{(s)}\right)$ to be the associated graded in $\mathbf{G r}^{\mathrm{b}}\left(A^{\prime}\right)$. This object can be made explicit: $\Gamma / \Delta$ is procyclic, say topologically generated by the image of $\gamma \in \Gamma$, and $\mathbf{R} \Gamma_{\text {cont }}\left(G, D^{(s)}\right)$ is represented by the complex

$$
\mathrm{C}_{\varphi, \gamma}^{\bullet}:\left[\left(D^{(s)}\right)^{\Delta} \stackrel{(\varphi-1, \gamma-1)}{\longrightarrow}\left(D^{(p s)}\right)^{\Delta} \oplus\left(D^{(s)}\right)^{\Delta} \stackrel{(1-\gamma, \varphi-1)}{\longrightarrow}\left(D^{(p s)}\right)^{\Delta}\right]
$$

in $\mathbf{K}^{\mathrm{b}}\left(A^{\prime}\right)$ concentrated in degrees 0,1 , and 2 .

Remark 2.4. It is easy to check that $\mathrm{H}^{i}\left(G, \operatorname{Hom}_{\mathrm{B}^{(s)}}\left(D^{(s)}, D^{\prime(s)}\right)\right)$ computes the Yoneda group $\operatorname{Ext}_{\mathbf{M}(\varphi, \Gamma)_{/ \mathbf{B}}^{(s)}}^{i}\left(D^{(s)}, D^{\prime(s)}\right)$, where $D^{(s)}$ and $D^{\prime(s)}$ are any two $(\varphi, \Gamma)$ modules over $\mathrm{B}^{(s)}$, for $i \leq 1$.

For two $(\varphi, \Gamma)$-modules $D^{(s)}$ and $D^{\prime(s)}$ over $\mathrm{B}^{(s)}$, we define cup products on cochains as in [Liu 2007]. In the representation $\mathrm{C}_{\varphi, \gamma}^{\bullet}$, the map

$$
\mathrm{C}_{\varphi, \gamma}^{i}\left(D^{(s)}\right) \otimes_{A^{\prime}} \mathrm{C}_{\varphi, \gamma}^{j}\left(D^{\prime(s)}\right) \rightarrow \mathrm{C}_{\varphi, \gamma}^{i+j}\left(D^{(s)} \otimes_{\mathrm{B}^{(s)}} D^{\prime(s)}\right)
$$

is the obvious multiplication when $i=0$ or $j=0$, and otherwise, we have

$$
\begin{aligned}
\mathrm{C}_{\varphi, \gamma}^{1}\left(D^{(s)}\right) \otimes_{A^{\prime}} \mathrm{C}_{\varphi, \gamma}^{1}\left(D^{\prime(s)}\right) & \rightarrow \mathrm{C}_{\varphi, \gamma}^{2}\left(D^{(s)} \otimes_{\mathrm{B}^{(s)}} D^{\prime(s)}\right), \\
\left(d_{1}, d_{2}\right) \otimes\left(d_{1}^{\prime}, d_{2}^{\prime}\right) & \mapsto d_{2} \otimes \gamma\left(d_{1}^{\prime}\right)-d_{1} \otimes \varphi\left(d_{2}^{\prime}\right) .
\end{aligned}
$$


The finite generation of the Galois cohomology of $(\varphi, \Gamma)$-modules is now known thanks to [Kedlaya et al. 2012], which appeared after the writing of this paper. We state the result here and henceforth refer to it as the finiteness theorem when it is invoked. We stress that $K / \mathbf{Q}_{p}$ is assumed to be finite.

Theorem 2.5. For the $(\varphi, \Gamma)$-module D over $A^{\prime} \widehat{\otimes}_{\mathbf{Q}_{p}} \mathrm{~B}_{\mathrm{rig}, K}^{\dagger}$, the Galois cohomology $\mathbf{R} \Gamma(G, D)$ belongs to $\mathbf{D}_{\text {perf }}^{[0,2]}\left(A^{\prime}\right)$, and the morphism

$$
\mathbf{R} \Gamma\left(G, D^{*}(1)\right) \rightarrow \mathbf{R} \Gamma(G, D)^{*}[-2]
$$

adjoint to the pairing given by cup product and evaluation, comparison (see Theorem 2.8 below) and truncation, and the local trace map, namely

$$
\begin{aligned}
\mathbf{R} \Gamma\left(G, D^{*}(1)\right) \otimes_{A^{\prime}}^{\mathbf{L}} \mathbf{R} \Gamma(G, D) \rightarrow \mathbf{R} \Gamma\left(G, A^{\prime} \widehat{\otimes}_{\mathbf{Q}_{p}}\right. & \left.\mathbf{B}_{\mathrm{rig}, K}^{\dagger}(1)\right) \\
& \rightarrow \tau_{\geq 2} \mathbf{R} \Gamma_{\mathrm{cont}}\left(G, A^{\prime}(1)\right) \cong A^{\prime},
\end{aligned}
$$

is an isomorphism.

The following proposition is proved without using the finiteness theorem and in fact is an ingredient in its proof:

Proposition 2.6. Let $D^{(s)}$ be a $(\varphi, \Gamma)$-module over $\mathrm{B}^{(s)}$.

(1) $D^{(s)}$ is a flat $A^{\prime}$-module.

(2) Suppose $B^{\prime}$ is an affinoid $A^{\prime}$-algebra. Then the natural map in $\mathbf{D}^{b}\left(B^{\prime}\right)$

$$
\mathbf{R} \Gamma\left(G, D^{(s)}\right) \otimes_{A^{\prime}}^{\mathbf{L}} B^{\prime} \rightarrow \mathbf{R} \Gamma\left(G, D^{(s)} \widehat{\otimes}_{A^{\prime}} B^{\prime}\right)
$$

is an isomorphism if $B^{\prime}$ is a finite $A^{\prime}$ algebra or if the modules $\mathrm{H}^{*}\left(G, D^{(s)}\right)$ and $\mathrm{H}^{*}\left(G, D^{(s)} \widehat{\otimes}_{A^{\prime}} B^{\prime}\right)$ are finitely generated over $A^{\prime}$ and $B^{\prime}$, respectively.

Proof. Since $D^{(s)}$ is a projective $\mathrm{B}^{(s)}$-module and each appropriate ring $\mathrm{B}^{(s)}$ is a flat $A^{\prime}$-algebra, (1) follows.

For (2), we use (1) to identify our map with

$$
h:\left[\mathrm{C}_{\varphi, \gamma}^{\bullet}\left(D^{(s)}\right) \otimes_{A^{\prime}} B^{\prime}\right] \rightarrow\left[\mathrm{C}_{\varphi, \gamma}^{\bullet}\left(D^{(s)}\right) \widehat{\otimes}_{A^{\prime}} B^{\prime}\right] .
$$

Under the first condition, we have $\otimes_{A^{\prime}} B^{\prime}=\widehat{\otimes}_{A^{\prime}} B^{\prime}$, so the result is trivial. Under the second condition, each $\mathrm{H}^{i}(h)$ is a map of finitely generated $B^{\prime}$-modules, so it suffices to show that the induced map $\mathrm{H}^{i}(h) \otimes_{B^{\prime}} B^{\prime} / \mathfrak{m}^{n}$ is an isomorphism for each maximal ideal $\mathfrak{m} \subset B$ and $n \geq 0$. One has a morphism of spectral sequences

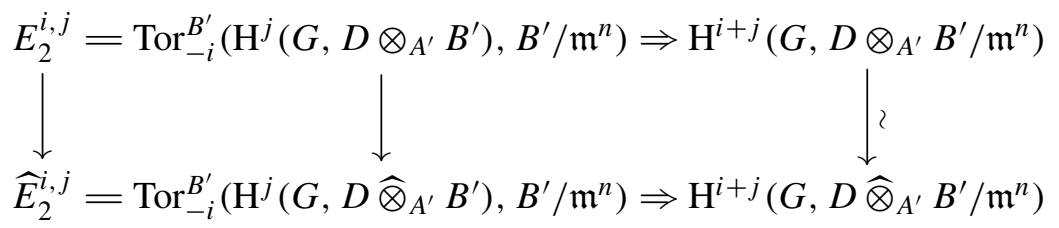


where the downward isomorphisms on the abutments are due to the fact that $B^{\prime} / \mathfrak{m}^{n}$ is a finite $A^{\prime}$-algebra. Using that $\mathrm{H}^{j}=0$ for $j>2$ throughout, one deduces immediately that $\mathrm{H}^{2}(h)$ is an isomorphism and then proceeds by repeated application of the five lemma to show that $\mathrm{H}^{1}(h)$, and then $\mathrm{H}^{0}(h)$, are isomorphisms.

Given a finite Galois extension $L / K$ inside $K^{\text {alg }}$, for any $(\varphi, \Gamma)$-module $D^{(s)}$, we leave it to the reader to define restriction and corestriction maps

$$
\begin{aligned}
\operatorname{res}_{L / K}: \mathbf{R} \Gamma\left(G_{K}, D^{(s)}\right) & \rightarrow \mathbf{R} \Gamma\left(G_{L}, D_{L}^{(s)}\right), \\
\operatorname{cores}_{L / K}: \mathbf{R} \Gamma\left(G_{L}, D_{L}^{(s)}\right) & \rightarrow \mathbf{R} \Gamma\left(G_{K}, D^{(s)}\right),
\end{aligned}
$$

whose composition cores ${ }_{L / K} \circ \operatorname{res}_{L / K}$ induces multiplication by $[L: K]$ on cohomology. It follows that $\mathrm{H}^{*}\left(G_{K}, D^{(s)}\right)$ is functorially a direct summand of $\mathrm{H}^{*}\left(G_{K}, D_{L}^{(s)}\right)$ and that, when $\mathrm{B}^{(s)}=A^{\prime} \widehat{\otimes}_{\mathbf{Q}_{p}} \mathrm{~B}_{K}^{\dagger(, s)}$, this decomposition is respected by the maps induced by $D^{(s)} \rightarrow \widetilde{D}^{\dagger(, s)}$ and $D^{(s)} \rightarrow D_{\text {rig }}^{\dagger(, s)}$.

If $X$ is a $p$-adic analytic space over $E$ and if $D$ is a family of $(\varphi, \Gamma)$-modules over $X$ of type $\mathrm{B}_{\text {rig, } K}^{\dagger}$, then by the finiteness theorem and Proposition 2.6(2), the rule $\Gamma\left(Y, \mathrm{C}_{\varphi, \gamma}^{\bullet}(D)\right)=\mathrm{C}_{\varphi, \gamma}^{\bullet}(\Gamma(Y, D))$ determines an object $\mathrm{C}_{\varphi, \gamma}^{\bullet}(D)$ of $\mathbf{K}_{\mathrm{ft}}^{\mathrm{b}}(\mathcal{U})$, whose class $\mathbf{R} \Gamma(G, D)$ in the derived category belongs to $\mathbf{D}_{\text {perf }}^{[0,2]}(\mathcal{\varphi})$.

2C. Galois cohomology of Galois representations. Throughout this section, let $M \in \operatorname{Rep}_{A^{\prime}}(G)$, and assume that $A^{\prime}$ is an affinoid algebra.

Proposition 2.7. The natural maps

$$
\begin{aligned}
\mathbf{R} \Gamma\left(G, \mathbf{D}^{\dagger}(M)\right) & \rightarrow \mathbf{R} \Gamma\left(G, \widetilde{\mathbf{D}}^{\dagger}(M)\right), \\
\mathbf{R} \Gamma\left(G, \mathbf{D}^{\dagger(, s)}(M)\right) & \rightarrow \mathbf{R} \Gamma\left(G, \mathbf{D}_{\text {rig }}^{\dagger(, s)}(M)\right)
\end{aligned}
$$

are isomorphisms in $\mathbf{D}^{\mathrm{b}}\left(A^{\prime}\right)$.

Proof. In order to check whether the induced maps on cohomology are isomorphisms, it suffices to check whether they become isomorphisms when restricted to the members of an affinoid covering of $A^{\prime}$. Thus, we reduce to the case where $M$ is free over $A^{\prime}$. By replacing $A$ by a different unit ball subalgebra of $A^{\prime}$, we may assume that $M=M_{0}[1 / p]$ for a finitely generated, free $A$-lattice $M_{0}$ that is $G$ stable. Choose a finite Galois extension $L / K$ inside $K^{\text {alg }}$ such that $G_{L}$ acts trivially on $M_{0} / 12 p$. Since the morphisms in question respect the direct sum decompositions of the Galois cohomology over $L$ coming from inflation and restriction relative to $L / K$, it suffices to prove the theorem with $K$ replaced by $L$, and thus, we may assume that $\mathbf{D}^{\dagger, s}(M)$ is a free module.

Consider the first map. It suffices to show that the natural morphism

$$
\mathbf{R} \Gamma_{\text {cont }}\left(\Gamma, \mathbf{D}^{\dagger}(M)\right) \rightarrow \mathbf{R} \Gamma_{\text {cont }}\left(\Gamma, \widetilde{\mathbf{D}}^{\dagger}(M)\right)
$$


is an isomorphism in $\mathbf{D}(A)$. A standard fact in the Tate-Sen theory of $(\varphi, \Gamma)$ modules is that $\widetilde{\mathbf{D}}^{\dagger}(M)$ admits a Galois-stable topological $\left(A \widehat{\otimes} \mathbf{z}_{p} \mathbf{B}_{K}^{\dagger}\right)$-direct sum decomposition as $\mathbf{D}^{\dagger}(M) \oplus X$ such that $\gamma-1$ acts bijectively on $X$ with continuous inverse. (See [Andreatta and Iovita 2008, Theorem 7.16] for an explanation of the method, taking $d=0$ everywhere, and generalize it to $A$-valued $M$ as in [Berger and Colmez $2008, \S 3]$.) Since $\mathbf{R} \Gamma_{\text {cont }}(\Gamma, X) \cong 0$, the claim follows.

For the second map, in the case with superscripts, one copies the proof of [Kedlaya 2008, Proposition 1.2.6] verbatim to obtain that the natural morphism of complexes

$$
\left[\mathbf{D}^{\dagger, s}(M) \stackrel{\varphi-1}{\longrightarrow} \mathbf{D}^{\dagger, p s}(M)\right] \rightarrow\left[\mathbf{D}_{\text {rig }}^{\dagger, s}(M) \stackrel{\varphi-1}{\longrightarrow} \mathbf{D}_{\text {rig }}^{\dagger, p s}(M)\right]
$$

is a quasi-isomorphism; the claim follows from this and the definitions. One obtains the case without superscripts from the former by taking $\lim _{\rightarrow}$.

When $A$ is a finite $\mathbf{Z}_{p}$-algebra, the following main result is due to Liu [2007]:

Theorem 2.8. There is a functorial isomorphism

$$
\mathbf{R} \Gamma_{\text {cont }}(G, M) \stackrel{\sim}{\rightarrow} \mathbf{R} \Gamma(G, \mathbf{D}(M))
$$

in $\mathbf{D}^{\mathrm{b}}\left(A^{\prime}\right)$, which is compatible with cup products and in degrees $i \leq 1$ agrees with applying $\mathbf{D}$ to Yoneda extension classes.

The key to the proof is the following:

Lemma 2.9. The obvious maps

$$
\begin{aligned}
& A^{\prime} \rightarrow \operatorname{Cone}\left[A^{\prime} \widehat{\otimes}_{\mathbf{Q}_{p}} \widetilde{\mathrm{B}}^{\dagger, s} \stackrel{\varphi-1}{\longrightarrow} A^{\prime} \widehat{\otimes}_{\mathbf{Q}_{p}} \widetilde{\mathrm{B}}^{\dagger, p s}\right][-1], \\
& A^{\prime} \widehat{\otimes} \mathbf{Q}_{p} \widetilde{\mathrm{B}}_{K}^{\dagger, s} \rightarrow \mathbf{R} \Gamma_{\text {cont }}\left(H, A^{\prime} \widehat{\otimes} \mathbf{Q}_{p} \widetilde{\mathrm{B}}^{\dagger, s}\right)
\end{aligned}
$$

are isomorphisms in $\mathbf{D}\left(A^{\prime}\right)$.

Proof. In the case $A^{\prime}=\mathbf{Q}_{p}$, the lemma is well-known, so we have exact sequences

$$
\begin{aligned}
& 0 \rightarrow \mathbf{Q}_{p} \rightarrow \widetilde{\mathrm{B}}^{\dagger, s} \stackrel{\varphi-1}{\longrightarrow} \widetilde{\mathrm{B}}^{\dagger, p s} \rightarrow 0, \\
& 0 \rightarrow \widetilde{\mathrm{B}}_{K}^{\dagger, s} \rightarrow \mathrm{C}_{\text {cont }}^{0}\left(H, \widetilde{\mathrm{B}}^{\dagger, s}\right) \rightarrow \mathrm{C}_{\text {cont }}^{1}\left(H, \widetilde{\mathrm{B}}^{\dagger, s}\right) \rightarrow \cdots .
\end{aligned}
$$

To deduce the result, we simply note that the functor $\widehat{\otimes}_{\mathbf{Q}_{p}} S$ preserves exact sequences of $\mathbf{Q}_{p}$-Banach spaces whenever $S$ is potentially orthonormalizable in the sense of [Buzzard 2007, §2] (even though this functor does not commute with formation of cohomology in general) and that any affinoid algebra has the latter property.

Proof of Theorem 2.8. By Proposition 2.7, it suffices to give a functorial isomorphism

$$
\mathbf{R} \Gamma_{\text {cont }}(G, M) \rightarrow \mathbf{R} \Gamma\left(G, \widetilde{\mathbf{D}}^{\dagger, s}(M)\right) .
$$


The compatibility with cup products and operations on Yoneda extensions follows from a routine trace through the definitions and hence is omitted.

It is easy to deduce from the preceding lemma a canonical isomorphism

$$
\mathbf{R} \Gamma_{\text {cont }}(H, M) \cong \operatorname{Cone}\left[\widetilde{\mathbf{D}}^{\dagger, s}(M) \stackrel{\varphi-1}{\longrightarrow} \widetilde{\mathbf{D}}^{\dagger, p s}(M)\right][-1] .
$$

Combining this isomorphism with a standard argument involving the HochschildSerre spectral sequence, the desired result follows.

We may now shed some light on the essential image of $\mathbf{D}_{\text {rig }}^{\dagger}$ on families of Galois representations, which at present is mysterious.

Corollary 2.10. Let $0 \rightarrow D^{\prime} \rightarrow E \rightarrow D \rightarrow 0$ be a short exact sequence of $(\varphi, \Gamma)$-modules over $A^{\prime} \widehat{\otimes}_{\mathbf{Q}_{p}} \mathrm{~B}_{\mathrm{rig}, K}^{\dagger, s}$. If $D$ and $D^{\prime}$ arise from $A^{\prime}$-valued Galois representations, then so does $E$.

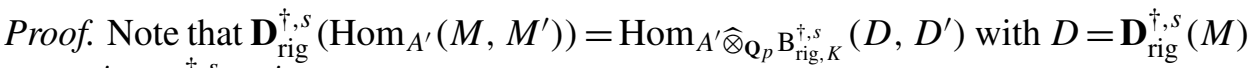
and $D^{\prime}=\mathbf{D}_{\text {rig }}^{\dagger, s}\left(M^{\prime}\right)$. By Remarks 1.8 and 2.4, to see the claim, it suffices to apply Theorem 2.8 to $\operatorname{Hom}_{A^{\prime}}\left(M, M^{\prime}\right)$ and take $\mathrm{H}^{1}$ of the result.

Let $X$ be a $p$-adic analytic space over $E$, let $M$ be a family of $G$-representations over $X$, and let $U$ be an admissible affinoid covering of $X$ that is quasiclosed under intersection. Then the functoriality of the isomorphisms in Theorem 2.8 gives rise to a canonical isomorphism $\mathbf{R} \Gamma_{\text {cont }}(G, M) \cong \mathbf{R} \Gamma(G, \mathbf{D}(M))$, which takes place in $\mathbf{D}_{\text {perf }}^{[0,2]}(U)$ by the finiteness theorem.

\section{Ordinary $(\varphi, \Gamma)$-modules and Selmer groups}

In this section, we put ourselves in the situation of Section 2, specializing to the case where $A^{\prime}=\mathbf{Q}_{p}$ and $\mathrm{B}^{(s)}=\mathrm{B}_{\mathrm{rig}, K}^{\dagger(, s)}$ unless otherwise specified.

3A. p-adic Hodge theory of $(\varphi, \Gamma)$-modules. This section describes the $p$-adic Hodge theory of $(\varphi, \Gamma)$-modules over the Robba ring. All the constructions and results to be found here are extensions of well-known ones for $p$-adic Galois representations, and many have explicitly appeared elsewhere; see, especially, works of Benois [2011] and Bellaïche and Chenevier [2009, §2.2]. The reader may check that every construction in this subsection holds with $K$ replaced by a general complete discretely valued field with the exception of the Euler-Poincare formula.

There exists a sequence of $\Gamma$-equivariant maps $\iota_{n}: \mathrm{B}_{\mathrm{rig}, K}^{\dagger, p^{n}} \rightarrow K_{n} \llbracket t \rrbracket$ for all $n \geq n(K)$, where $t$ is the element of $\mathrm{B}_{\text {rig, } K}^{\dagger}$ defined in Section $2 \mathrm{~A}$, such that $\iota_{n+1} \circ \varphi=\iota_{n}$. Given a $\varphi$-module $D$, we put $D_{\text {dif }}^{+}=D^{s} \otimes_{\mathrm{B}_{\text {rig, }, K}^{+, s}, \iota_{n}} K_{\infty} \llbracket t \rrbracket$, and $D_{\text {dif }}=D^{s} \otimes_{\mathrm{B}_{\mathrm{rig}, K}^{\dagger}, l_{n}}^{\dagger s} K_{\infty}((t))$, where $D^{s}$ is uniquely determined for $s>s(D)$ by [Berger 2008, Théorème I.3.3] and is a model of $D$ over $\mathrm{B}_{\text {rig, } K}^{\dagger, s}$. Using the $\varphi$ structure, one shows that these rules are independent of $s$ and $n$ satisfying $s>s(D)$ and $p^{n} \geq \max \left(p^{n(K)}, s\right)$. The rules $D \mapsto D_{\text {dif }}^{(+)}$are functorial and exact in $D$. 
If $D$ is actually a $(\varphi, \Gamma)$-module, then the $D_{\text {dif }}^{(+)}$admit $\Gamma$-actions (by perhaps enlarging the $s$ used), and we define $D_{\mathrm{dR}}^{(+)}=\left(D_{\mathrm{dif}}^{(+)}\right)^{\Gamma}$. These are $K$-vector spaces of dimension at most rank $D$, and they carry a decreasing, separated, and exhaustive filtration induced by the $t$-adic filtration on $K_{\infty}((t))$. One says that $D$ is de Rham if $\operatorname{dim}_{K} D_{\mathrm{dR}}=\operatorname{rank} D$ and denotes by $\mathbf{M}^{\mathrm{dR}}(\varphi, \Gamma) \subset \mathbf{M}(\varphi, \Gamma)$ the full subcategory of de Rham objects. For such $D$, we define its Hodge-Tate weights to be the $h \in \mathbf{Z}$ with $\mathrm{Gr}^{h} D_{\mathrm{dR}} \neq 0$ with respective multiplicities $\operatorname{dim}_{K} \mathrm{Gr}^{h} D_{\mathrm{dR}}$. (There is no standard convention for the sign of the Hodge-Tate weight of the cyclotomic character; in this paper, it is -1 .)

We write for brevity

$$
\begin{aligned}
D\left[t^{-1}\right] & =D \otimes_{\mathrm{B}_{\mathrm{rig}, K}^{\dagger}} \mathrm{B}_{\mathrm{rig}, K}^{\dagger}\left[t^{-1}\right], \\
D\left[\log \pi, t^{-1}\right] & =D \otimes_{\mathrm{B}_{\mathrm{rig}, K}^{\dagger}}^{\dagger} \mathrm{B}_{\mathrm{rig}, K}^{\dagger}\left[\log \pi, t^{-1}\right],
\end{aligned}
$$

where the element $\log \pi$ is a free variable over $\mathrm{B}_{\mathrm{rig}, K}^{\dagger}$ equipped with actions of $\varphi$ and $\Gamma$ by the formulas

$$
\varphi(\log \pi)=p \log \pi+\log \left(\varphi(\pi) / \pi^{p}\right) \quad \text { and } \quad \gamma(\log \pi)=\log \pi+\log (\gamma(\pi) / \pi),
$$

the series $\log \left(\varphi(\pi) / \pi^{p}\right)$ and $\log (\gamma(\pi) / \pi)$ being convergent in $\mathrm{B}_{\mathrm{rig}, \mathbf{Q}_{p}}^{\dagger}$. We associate to $D$ the modules

$$
D_{\text {crys }}^{+}=D^{\Gamma}, \quad D_{\text {crys }}=D\left[t^{-1}\right]^{\Gamma}, \quad \text { and } \quad D_{\text {st }}=D\left[\log \pi, t^{-1}\right]^{\Gamma} .
$$

These three modules are semilinear $\varphi$-modules over $F$ of dimension at most rank $D$. The latter two are related via the so-called monodromy operator $N$. Namely, consider the unique $\mathrm{B}_{\text {rig, } K}^{\dagger}$-derivation $N: \mathrm{B}_{\text {rig, } K}^{\dagger}[\log \pi] \rightarrow \mathrm{B}_{\text {rig, } K}^{\dagger}[\log \pi]$ satisfying $N(\log \pi)=-\frac{p}{p-1}$. It satisfies $N \varphi=p \varphi N$ and commutes with $\Gamma$ and thus gives rise to a nilpotent operator $N$ on $D_{\mathrm{st}}$ with the property that $D_{\text {crys }}=D_{\mathrm{st}}^{N=0}$.

We say that $D$ is crystalline or semistable if $D_{\text {crys }}$ or $D_{\text {st }}$ has the maximal $F$ dimension, namely $\operatorname{dim}_{F} D_{\text {crys }}=\operatorname{rank} D$ or $\operatorname{dim}_{F} D_{\text {st }}=\operatorname{rank} D$, respectively. Upon fixing a uniformizer for $K$, we can construct a canonical embedding $D_{\mathrm{st}} \otimes_{F} K \hookrightarrow D_{\mathrm{dR}}$ so that $D$ being semistable implies $D$ being de Rham. We call $D$ potentially crystalline or potentially semistable if there exists a finite extension $L / K$ inside $K^{\text {alg }}$ such that $D_{L}$ is crystalline or semistable, respectively, when considered as a $\left(\varphi, \Gamma_{L}\right)$-module. The following statement is known as Berger's $p$-adic local monodromy theorem:

Theorem 3.1 [Berger 2002]. Every de Rham $(\varphi, \Gamma)$-module is potentially semistable.

Given a de Rham $D$, let $L / K$ be a finite Galois extension inside $K^{\text {alg }}$ such that $D_{L}$ is semistable. Then $\left(D_{L}\right)_{\mathrm{st}}$ is a $(\varphi, N)$-module over the maximal absolutely unramified subfield $F_{L}$ of $L$, and $\left(D_{L}\right)_{\mathrm{st}} \otimes_{F_{L}} L=\left(D_{L}\right)_{\mathrm{dR}}$ is a filtered $L$-vector space. 
Essentially because these data arise via base change from $K$, they are naturally equipped with a semilinear action of $\operatorname{Gal}(L / K)$ that commutes with $\varphi$ and $N$ and preserves the filtration. Such an object is called a filtered $(\varphi, N, \operatorname{Gal}(L / K))$ module. Given two extensions $L_{i}$ and filtered $\left(\varphi, N, \operatorname{Gal}\left(L_{i} / K\right)\right)$-modules $D_{i}$ (for $i=1,2$ ), we consider them equivalent if there exists an extension $L$ containing the $L_{i}$ such that the $\left(D_{i}\right)_{L}$ are isomorphic. When we consider objects only up to this equivalence, we call them filtered $(\varphi, N, G)$-modules. We point out that if $D$ becomes semistable over both $L_{1}$ and $L_{2}$, then $\left(D_{L_{1}}\right)_{\mathrm{st}}$ and $\left(D_{L_{2}}\right)_{\mathrm{st}}$ are equivalent, and we call this equivalence class $D_{\text {pst }}$. The rigid exact $\mathbf{Q}_{p}$-linear tensor category of filtered $(\varphi, N, G)$-modules is denoted $\mathbf{M F}(\varphi, N, G)$.

The objects $M$ of the category $\mathbf{M F}(\varphi, N, G)$ admit a notion of degree, namely the Newton slope minus the Hodge-Tate weight of $M^{\wedge \operatorname{rank}(M)}$, which gives rise to a Harder-Narasimhan theory. One calls $M$ (weakly) admissible if it is semistable of slope 0 in the sense of Harder-Narasimhan theory. (See [Berger 2008, §I.1] for details.)

Theorem 3.2 [Colmez and Fontaine 2000; Berger 2008]. The functor $D \mapsto D_{\mathrm{pst}}$ is an exact equivalence of categories

$$
\mathbf{M}^{\mathrm{dR}}(\varphi, \Gamma) \stackrel{\sim}{\rightarrow} \mathbf{M F}(\varphi, N, G)
$$

that matches their Harder-Narasimhan theories. In particular, a de Rham $(\varphi, \Gamma)$ module $D$ is étale if and only if $D_{\mathrm{pst}}$ is (weakly) admissible.

Comparing notions of image and coimage, one deduces that the $t$-saturated $(\varphi, \Gamma)$ stable $\mathrm{B}_{\text {rig, } K}^{\dagger}$-submodules of $D$ are in a functorial, order-preserving correspondence with subspaces of $D_{\text {pst }}$ that are stable under the $(\varphi, N, G)$-actions (equipped with the filtration induced from $\left.D_{\mathrm{pst}}\right)$. Furthermore, a $t$-saturated $(\varphi, \Gamma)$-stable $\mathrm{B}_{\text {rig, } K^{-}}^{\dagger}$ submodule is actually a $\mathrm{B}_{\text {rig, } K}^{\dagger}$-direct summand.

The following immediate consequence of the $p$-adic monodromy theorem is usually stated for Galois representations, but the proof carries over without change for $(\varphi, \Gamma)$-modules. (As pointed out in [Berger 2002], the étale case was first proved by $\mathrm{O}$. Hyodo without use of the $p$-adic monodromy theorem, but the proof cited below works for arbitrary complete discretely valued $K$.)

Corollary 3.3 [Berger 2002, Théorème 6.2]. Let $0 \rightarrow D^{\prime} \rightarrow D \rightarrow D^{\prime \prime} \rightarrow 0$ be a short exact sequence of $(\varphi, \Gamma)$-modules. If $D^{\prime}$ and $D^{\prime \prime}$ are semistable and $D$ is de Rham, then $D$ is semistable.

As in Remark 2.4, the cohomology groups $\mathrm{H}^{1}(G, D)$ coincide with Yoneda groups: to every $c \in \mathrm{H}^{1}(G, D)$, there corresponds a class of extensions

$$
0 \rightarrow D \rightarrow E_{c} \rightarrow \mathbf{1} \rightarrow 0
$$


where 1 denotes the unit $(\varphi, \Gamma)$-module. The rule $\left[E_{c}\right] \mapsto\left[\left(E_{c}\right)_{\text {dif }}\right]$ determines a map $\mathrm{H}^{1}(G, D) \rightarrow \mathrm{H}_{\text {cont }}^{1}\left(\Gamma, D_{\text {dif }}\right)$, and the Bloch-Kato " $g$ " local subspace is given by

$$
\mathrm{H}_{\mathrm{g}}^{1}(G, D)=\operatorname{ker}\left[\mathrm{H}^{1}(G, D) \rightarrow \mathrm{H}_{\text {cont }}^{1}\left(\Gamma, D_{\text {dif }}\right)\right] .
$$

When $D$ is de Rham, one has

$$
\begin{aligned}
\mathrm{H}_{\mathrm{g}}^{1}(G, D) & =\left\{c \in \mathrm{H}^{1}(G, D) \mid E_{c} \text { is de Rham }\right\} \\
& =\left\{c \in \mathrm{H}^{1}(G, D) \mid E_{c} \text { is potentially semistable }\right\} \\
& =\operatorname{Ext}_{\mathbf{M F}(\varphi, N, G)}^{1}\left(\mathbf{1}, D_{\mathrm{pst}}\right) .
\end{aligned}
$$

Similarly, a map $\mathrm{H}^{1}(G, D) \rightarrow \mathrm{H}_{\text {cont }}^{1}(\Gamma, D[1 / t])$ is determined by forgetting $\varphi$-structures and inverting $t$, and we define the Bloch-Kato " $f$ " local subspace to be

$$
\mathrm{H}_{\mathrm{f}}^{1}(G, D)=\operatorname{ker}\left[\mathrm{H}^{1}(G, D) \rightarrow \mathrm{H}_{\text {cont }}^{1}\left(\Gamma, D\left[t^{-1}\right]\right)\right] .
$$

When $D$ is crystalline, one has

$$
\mathrm{H}_{\mathrm{f}}^{1}(G, D)=\left\{c \in \mathrm{H}^{1}(G, D) \mid E_{c} \text { is crystalline }\right\} .
$$

If $D$ is de Rham, then under the isomorphism (3-1) one can compute $\mathrm{H}_{\mathrm{f}}^{1}(G, D)$ as certain extensions of filtered $(\varphi, N, G)$-modules, obtaining the exact sequence

$$
0 \rightarrow \mathrm{H}^{0}(G, D) \rightarrow D_{\text {crys }} \stackrel{(1-\varphi, 1)}{\longrightarrow} D_{\text {crys }} \oplus D_{\mathrm{dR}} / D_{\mathrm{dR}}^{+} \rightarrow \mathrm{H}_{\mathrm{f}}^{1}(G, D) \rightarrow 0 .
$$

This computation can be enhanced to show that the local condition associated to the subspace $\mathrm{H}_{\mathrm{f}}^{1}(G, D)$ is isomorphic in the derived category to the complex

$$
\begin{aligned}
\mathrm{C}_{\mathrm{f}}^{\bullet}(G, D) & =\text { Cone }\left[D_{\text {crys }} \stackrel{(1-\varphi, 1)}{\longrightarrow} D_{\text {crys }} \oplus D_{\mathrm{dR}} / D_{\mathrm{dR}}^{+}\right][-1], \\
\mathbf{R} \Gamma_{\mathrm{f}}(G, D) & =\left[\mathrm{C}_{\mathrm{f}}^{\bullet}(G, D)\right],
\end{aligned}
$$

and one obtains the "Euler-Poincaré" formula

$$
\operatorname{dim}_{\mathbf{Q}_{p}} \mathrm{H}_{\mathrm{f}}^{1}(G, D)=\operatorname{dim}_{\mathbf{Q}_{p}} \mathrm{H}^{0}(G, D)+\operatorname{dim}_{\mathbf{Q}_{p}} D_{\mathrm{dR}} / D_{\mathrm{dR}}^{+} .
$$

The next result follows from the (elementary) computation of $\Gamma$-cohomology of $t^{n} K_{\infty} \llbracket t \rrbracket$ and that $K_{\infty} \llbracket t \rrbracket$ is a PID.

Proposition 3.4. Let $0 \rightarrow D^{\prime} \rightarrow D \rightarrow D^{\prime \prime} \rightarrow 0$ be a short exact sequence of $(\varphi, \Gamma)$-modules with $D^{\prime}$ and $D^{\prime \prime}$ de Rham. If all the Hodge-Tate weights of $D^{\prime}$ are strictly less than all the Hodge-Tate weights of $D^{\prime \prime}$, then the short exact sequence of $\Gamma$-modules

$$
0 \rightarrow\left(D^{\prime}\right)_{\mathrm{dif}}^{+} \rightarrow D_{\mathrm{dif}}^{+} \rightarrow\left(D^{\prime \prime}\right)_{\mathrm{dif}}^{+} \rightarrow 0
$$

is split. In particular, D is de Rham. 
3B. The (strict) ordinary local condition. We briefly relax our hypothesis that $A^{\prime}=\mathbf{Q}_{p}$; instead, $A^{\prime}$ can be any $E$-affinoid algebra.

Let $D$ be a $(\varphi, \Gamma)$-module over $A^{\prime} \widehat{\otimes}_{\mathbf{Q}_{p}} \mathrm{~B}_{\text {rig, } K}^{\dagger}$, or a family of $(\varphi, \Gamma)$-modules of type $\mathrm{B}_{\text {rig, } K}^{\dagger}$ over a $p$-adic analytic space $X$, endowed with an admissible affinoid covering $\mathcal{U}$ that is quasiclosed under intersection. By a nearly ordinary filtration on $D$, we mean a decreasing partial flag $F^{*} \subseteq D$, consisting of sub- $(\varphi, \Gamma)$-modules that are module-direct summands, or subfamilies of $(\varphi, \Gamma)$-modules that are direct summands over each $Y \in \mathcal{U}$, respectively, such that each $\mathrm{Gr}_{F}^{\alpha}$ has constant rank.

If $M$ is either an object of $\operatorname{Rep}_{A^{\prime}}(G)$ or a family of $G$-representations over $X$, then a nearly ordinary filtration for $M$ is by definition one for $\mathbf{D}_{\text {rig }}^{\dagger}(M)$, and we call it classically nearly ordinary if it arises from a partial flag of $M$ consisting of $G$-stable direct summands.

Example 3.5. When $A^{\prime}=\mathbf{Q}_{p}$, being nearly ordinary with a complete flag means being (split) trianguline.

Given a sub- $(\varphi, \Gamma)$-module $F^{+} \subseteq D$ that is a module-direct summand or a subfamily of $(\varphi, \Gamma)$-modules that are module-direct summands over each $Y \in \mathcal{U}$, we recall from Example 1.18 the (strict) ordinary local condition given by the morphism

$$
\mathbf{R} \Gamma_{\text {str }}(G, D)=\mathbf{R} \Gamma\left(G, F^{+}\right) \rightarrow \mathbf{R} \Gamma(G, D) .
$$

In the case $D=\mathbf{D}_{\text {rig }}^{\dagger}(M)$, we get the local condition for $M$,

$$
\mathbf{R} \Gamma_{\text {str }}(G, D)=\mathbf{R} \Gamma\left(G, F^{+}\right) \rightarrow \mathbf{R} \Gamma(G, D) \cong \mathbf{R} \Gamma_{\text {cont }}(G, M) .
$$

By the finiteness theorem, both the domain and codomain belong to $\mathbf{D}_{\text {perf }}^{[0,2]}\left(A^{\prime}\right)$, and the formation of this local condition commutes with arbitrary base change by Proposition 2.6(2) as well as with duality (in the same sense as does the classical strict ordinary local condition as in Example 1.18) again by the finiteness theorem. The image of the local condition in cohomology is clearly

$$
\operatorname{img}\left[\mathrm{H}^{1}\left(G, F^{+}\right) \rightarrow \mathrm{H}^{1}(G, D)\right]=\operatorname{ker}\left[\mathrm{H}^{1}(G, D) \rightarrow \mathrm{H}^{1}\left(G, D / F^{+}\right)\right],
$$

which is a generalization of the (strict) ordinary local subspace studied by Greenberg [1994b] in conjunction with the nonstrict ordinary local subspace (introduced earlier in [Greenberg 1989; 1994a])

$$
\operatorname{ker}\left[\mathrm{H}^{1}\left(G_{K}, D\right) \rightarrow \mathrm{H}^{1}\left(G_{\widehat{K^{u n r}}},\left(D / F^{+}\right) \otimes_{\mathrm{B}_{\mathrm{rig}, K}^{\dagger}} \mathrm{B}_{\mathrm{rig}, \widehat{K^{\text {unr }}}}^{\dagger}\right)\right] .
$$

Although the nonstrict local subspace appears more often in the literature, we will not use it essentially because a derived analogue, like $\mathbf{R} \Gamma_{\text {str }}(G, D)$ in the strict case, would involve Galois cohomology for the group $G_{\widehat{K}}$ unr , which does not satisfy $p$-cohomological finiteness, rendering the derived analogue pathological. 
We now resume our assumption that $A^{\prime}=\mathbf{Q}_{p}$.

Let $L / K$ be a finite Galois extension inside $K^{\text {alg }}$. We say a $(\varphi, \Gamma)$-module $D$ over $\mathrm{B}_{\mathrm{rig}, K}^{\dagger}$ is $L$-ordinary if it admits a nearly ordinary flag $F^{*} \subseteq D$ with the properties that each $\left(\mathrm{Gr}_{F}^{\alpha}\right)_{L}$ is semistable and if $\alpha<\beta$ then all Hodge-Tate weights of $\mathrm{Gr}_{F}^{\alpha}$ are strictly greater than all Hodge-Tate weights of $\mathrm{Gr}_{F}^{\beta}$. We say that $D$ is ordinary if there exists some $L$ for which it is $L$-ordinary. If $V$ is a Galois representation, we say that $V$ is ( $L$-)ordinary if $\mathbf{D}_{\text {rig }}^{\dagger}(V)$ is and classically $(L$-)ordinary if there exists a partial flag of $V$ by $G$-stable direct summands giving rise via $\mathbf{D}_{\text {rig }}^{\dagger}$ to the desired filtration.

Example 3.6. We will see in Section 3C that Greenberg's notion of "ordinary" translates to our classically $K$-ordinary with each $\mathrm{Gr}_{F}^{\alpha}$ having only one Hodge-Tate weight (possibly with multiplicity). Thereafter, we will see examples of ordinary but not classically ordinary Galois representations. It is possible that $D$ be ordinary with respect to more than one filtration even though ordinary filtrations in Greenberg's sense are unique when they exist.

Here are the main properties of ordinary $(\varphi, \Gamma)$-modules:

Proposition 3.7. Let $L / K$ be a finite Galois extension inside $K^{\mathrm{alg}}$, and let $D$ be a $(\varphi, \Gamma)$-module that is L-ordinary with filtration $F^{*}$.

(1) D becomes semistable over $L$ and hence is potentially semistable.

Suppose moreover that there exists a filtration step $F^{+}$with the property that all the Hodge-Tate weights of $\mathrm{F}^{+}$are negative and all the Hodge-Tate weights of $D / F^{+}$ are nonnegative.

(2) One has $\mathrm{H}^{0}\left(G, D / F^{+}\right)=\left(D / F^{+}\right)_{\text {crys }}^{\varphi=1}$ and $\mathrm{H}^{0}\left(G,\left(F^{+}\right)^{*}(1)\right)=\left(\left(F^{+}\right)^{*}(1)\right)_{\text {crys }}^{\varphi=1}$.

(3) Suppose that all the spaces mentioned in part (2) vanish. Then the canonical maps in the derived category are isomorphisms:

$$
\mathbf{R} \Gamma\left(G, F^{+}\right) \underset{\leftarrow}{ } \mathbf{R} \Gamma_{\mathrm{f}}\left(G, F^{+}\right) \stackrel{\sim}{\rightarrow} \mathbf{R} \Gamma_{\mathrm{f}}(G, D),
$$

hence the local conditions

$$
\mathbf{R} \Gamma_{\mathrm{str}}(G, D) \cong \mathbf{R} \Gamma_{\mathrm{f}}(G, D) \quad \text { and } \quad \mathrm{H}^{1}\left(G, F^{+}\right) \hookrightarrow \mathrm{H}^{1}(G, D) .
$$

Remark 3.8. A variant of the proposition can be formulated for general $K$ complete discretely valued, but we omit it for brevity. Suppose $D=\mathbf{D}_{\text {rig }}^{\dagger}(V)$ with $V$ ordinary in the sense of Greenberg. When $K / \mathbf{Q}_{p}$ is finite, the claim (1) for $V$ is due to Fontaine [Perrin-Riou 1994a], and for general $K$, it is due to Berger [2002, Corollaire 6.3]. When $K=\mathbf{Q}_{p}$, the claim (3) for $V$ is essentially a result of Flach [1990, Lemma 2]. Our formulation of parts (2)-(3) closely follows that of Fukaya and Kato [2006, Lemma 4.1.7]. 
Proof. For part (1), by restriction, we immediately reduce to the case where $D$ is $K$ ordinary. The first claim now follows by induction on the length of the filtration, the case of length 1 being trivial and the inductive step being given by Proposition 3.4 and Corollary 3.3.

Part (2) follows from noting that both $D / F$ and $F^{*}(1)$ have only nonnegative Hodge-Tate weights and applying to them the claim that for any $(\varphi, \Gamma)$-module $D$ one has $\mathrm{H}^{0}(G, D)=D_{\text {crys }}^{+, \varphi=1}$.

We now turn to part (3). For the first arrow, we have equality of cohomology outside degrees 1 and 2. For degree 1, we compute that

$$
\begin{aligned}
\operatorname{dim}_{\mathbf{Q}_{p}} \mathrm{H}^{1}\left(G, F^{+}\right) & =\operatorname{dim}_{\mathbf{Q}_{p}} \mathrm{H}^{0}\left(G, F^{+}\right)+\operatorname{dim}_{\mathbf{Q}_{p}} \mathrm{H}^{2}\left(G, F^{+}\right)+\left[K: \mathbf{Q}_{p}\right] \operatorname{rank} F^{+} \\
& =\operatorname{dim}_{\mathbf{Q}_{p}} \mathrm{H}^{0}\left(G, F^{+}\right)+\operatorname{dim}_{\mathbf{Q}_{p}}\left(F^{+}\right)_{\mathrm{dR}} /\left(F^{+}\right)_{\mathrm{dR}}^{+} \\
& =\operatorname{dim}_{\mathbf{Q}_{p}} \mathrm{H}_{\mathrm{f}}^{1}\left(G, F^{+}\right),
\end{aligned}
$$

using the local Euler-Poincaré formulas of [Liu 2007] and Equation (3-2) for $F^{+}$, the computation $\mathrm{H}^{2}\left(G, F^{+}\right)=\mathrm{H}^{0}\left(G,\left(F^{+}\right)^{*}(1)\right)^{*}=0$ by local duality for $(\varphi, \Gamma)$ modules [Liu 2007], and that $F^{+}$has only negative Hodge-Tate weights. The same local duality computation takes care of degree 2 . For the second arrow, we have equality of cohomology outside degrees 0 and 1 . The long exact cohomology sequence and the vanishing of $\mathrm{H}^{0}\left(G, D / F^{+}\right)$give the result in degree 0 and the injectivity of $\mathrm{H}^{1}\left(G, F^{+}\right) \rightarrow \mathrm{H}^{1}(G, D)$ and hence also of $\mathrm{H}_{\mathrm{f}}^{1}\left(G, F^{+}\right) \rightarrow \mathrm{H}_{\mathrm{f}}^{1}(G, D)$. To conclude in degree 1, it suffices to compare the Euler-Poincare formulas for the two dimensions, noting that $\left(F^{+}\right)_{\mathrm{dR}} /\left(F^{+}\right)_{\mathrm{dR}}^{+} \underset{\leftarrow}{\leftarrow}\left(F^{+}\right)_{\mathrm{dR}} \stackrel{\sim}{\rightarrow} D_{\mathrm{dR}} / D_{\mathrm{dR}}^{+}$.

3C. Examples of ordinary representations. When discussing examples, the following equivalent formulation is helpful:

Alternate definition 3.9. We remind the reader that by Proposition 3.7(1), every ordinary $(\varphi, \Gamma)$-module is de Rham, so we assume this is the case from the outset.

Given a de Rham $(\varphi, \Gamma)$-module $D$, by the discussion of Section $3 \mathrm{~A}$, the ordinary filtrations $F^{*} \subseteq D$ are in a natural correspondence with filtrations $F^{*} \subseteq D_{\text {pst }}$ by $(\varphi, N, G)$-stable subspaces (each equipped with its Hodge filtration induced by $D_{\text {pst }}$ ) such that for $\alpha<\beta$ all the jump indices of the induced Hodge filtration on $\left(\mathrm{Gr}_{F}^{\alpha}\right)_{\mathrm{dR}}$ are strictly greater than all the jump indices of the induced Hodge filtration on $\left(\mathrm{Gr}_{F}^{\beta}\right)_{\mathrm{dR}}$. Note the reversal of order of the jump indices: this feature is independent of one's normalizations of Hodge-Tate weights.

Example 3.10 (Greenberg's ordinary representations). Let us see how ordinary representations, defined by Greenberg [1989] when $K=\mathbf{Q}_{p}$, fit into our context. We are given a Galois representation $V$ so that $D=\mathbf{D}_{\text {rig }}^{\dagger}(V)$ is étale. Greenberg's ordinary hypothesis is that $V$ admits a decreasing filtration $F^{*} \subseteq V$ by $G$-stable subspaces such that for each $\alpha$ the representation $\chi_{\text {cycl }}^{-n_{\alpha}} \otimes \mathrm{Gr}_{F}^{\alpha} V$ is unramified 
for some integer $n_{\alpha}$, and the $n_{\alpha}$ are strictly increasing. This means precisely that each $\operatorname{Gr}_{F}^{\alpha} V$ is crystalline of all Hodge-Tate weights equal to $-n_{\alpha}$. Thus, Greenberg's ordinary hypothesis is a strengthening of our classically $K$-ordinary hypothesis to require that each of the graded pieces be of a single Hodge-Tate weight. In the language of filtered $(\varphi, N, G)$-modules, a filtration $F^{*} \subseteq D_{\text {pst }}$ corresponds to a Greenberg-ordinary filtration on $V$ precisely when $V$ is semistable, and each $\mathrm{Gr}_{F}^{\alpha} D_{\mathrm{pst}}$ is (weakly) admissibly filtered of a single Hodge-Tate weight, with the weights strictly decreasing, which means here that each $\operatorname{Gr}_{F}^{\alpha} D_{\text {pst }}$ is of pure $\varphi$-slope $-n_{\alpha}$ and Hodge-Tate weight $-n_{\alpha}$ and satisfies $N=0$ and that $n_{\alpha}>n_{\beta}$ for $\alpha<\beta$.

The reader will notice in the examples below that although $V$ admits at most one Greenberg-ordinary filtration it may admit many different $(\varphi, \Gamma)$-ordinary filtrations. This is complementary to the existence of many $p$-adic L-functions.

Example 3.11 (Abelian varieties). Take an abelian variety $B / K$ of dimension $d \geq 1$ with semistable reduction over $0_{K}$, and consider $D=\mathbf{D}_{\text {rig }}^{\dagger}(V)$ with $V=\mathrm{T}_{p} B \otimes \mathbf{Q}$ the $p$-adic Tate module up to isogeny. The Hodge filtration Hodge* $\subseteq D_{\mathrm{dR}}$ satisfies $\operatorname{dim}_{K} \mathrm{Gr}_{\text {Hodge }}^{0}=\operatorname{dim}_{K} \mathrm{Gr}_{\text {Hodge }}^{-1}=d$, and its Frobenius slopes $h$ satisfy $-1 \leq h \leq 0$. By weak admissibility, the $\varphi$-eigenspaces with nonzero slopes do not meet Hodge ${ }^{0}$. A nontrivial ordinary filtration thus consists of a $(\varphi, N)$-stable subspace $F \subseteq D_{\text {st }}$ of rank $d$ such that $F_{\mathrm{dR}}$ is complementary to Hodge ${ }^{0}$ in $D_{\mathrm{dR}}$.

Example 3.12 (Elliptic modular eigenforms). This case is treated in detail in [Pottharst 2012], the upshot being as follows. Let $p>2$, and let $f$ be a normalized elliptic modular cuspidal new eigenform of weight $k \geq 2$ with associated cohomological $p$-adic Galois representation $V_{f}$. If necessary, extend the scalars of $V_{f}$ to contain the eigenvalues of $\varphi$ on $\mathbf{D}_{\mathrm{pst}}\left(V_{f}\right)$. Then $V_{f}$ is ordinary in our sense, often with two distinct ordinary filtrations, provided $f$ has finite slope: the matrix of $\varphi$ on $\mathbf{D}_{\mathrm{pst}}\left(V_{f}\right)$ is nonscalar. For example, when $\varphi$ on $\mathbf{D}_{\mathrm{pst}}\left(V_{f}\right)$ is semisimple (as is conjecturally always the case), this is equivalent to there being some twist $f \otimes \varepsilon$ by a Dirichlet character $\varepsilon$ that has an associated $U_{p}$-eigenform with nonzero $U_{p}$-eigenvalue. By contrast, $f$ is "ordinary" in the parlance of $p$-adic modular forms if this condition is satisfied with $\varepsilon=1$ and the $U_{p}$-eigenvalue a $p$-adic unit. Proposition 3.7(3) computes the Bloch-Kato local condition entering into the Selmer group of each of the Tate twists $V_{f}(n)$ corresponding to critical L-values except perhaps where exceptional zeroes (as in [Mazur et al. 1986]) occur.

3D. Ranks in families. We resume the assumptions of Section 1E, i.e., that $K$ is a finite extension of $\mathbf{Q}$, that $K^{\text {alg }}$ is a fixed algebraic closure, and that $S$ is a fixed finite set of places $v$ of $K$ containing all $v$ dividing $p$. Let $M$ be a family of $G_{K, S}$-representations over a $p$-adic analytic space $X$, endowed with an admissible affinoid covering $U$ that is quasiclosed under intersection. (For example, one can have $X$ affinoid with algebra $A^{\prime}$ and $M$ arising from a flat $A^{\prime}$-module.) Suppose 
that, for each place $v \in S$ dividing $p$, the restriction $\left.M\right|_{G_{v}}$ is equipped with a nearly ordinary filtration $F_{v}^{*} \subseteq D_{v}=\mathbf{D}_{\text {rig }}^{\dagger}\left(\left.M\right|_{G_{v}}\right)$, and we have a distinguished index $\alpha_{v}$.

In order to get a reasonable theory, we must assume that for each $v \in S$ not dividing $p$ the subobject $M^{I_{v}}$ is flat.

Using the strict ordinary local condition given by the $F_{v}^{\alpha_{v}}$ at places $v \in S$ dividing $p$ and the unramified local condition at places $v \in S$ not dividing $p$, we build as in situation (3) of Section 1E the strict ordinary Selmer complex, denoted

$$
\mathbf{R} \widetilde{\Gamma}_{\text {str }}\left(G_{K}, M\right) \in \mathbf{D}_{\text {perf }}^{[0,3]}(\mathcal{( U )} .
$$

One can check that it is invariant under enlarging $S$ and hence is independent of $S$, so we may omit it from the notation.

We wish to compare the above complex to those associated to the members of the family. Namely, let $x \in X\left(E_{x}\right)$ be a point with residue field $E_{x}$, and let $f_{x}$ denote the inclusion of the point $x$. We set $M_{x}=f_{x}^{*} M, D_{v, x}=f_{x}^{*} D_{v}$, and $F_{v, x}^{*}=f_{x}^{*} F_{v}^{*}$ for $v \in S$, and we use the strict ordinary local condition determined by the $F_{v, x}^{\alpha_{v}}$ at $v \in S$ above $p$, and the unramified local condition at $v \in S$ not dividing $p$, to construct in the same way the Selmer complex

$$
\mathbf{R} \widetilde{\Gamma}_{\text {str }}\left(G_{K}, M_{x}\right) \in \mathbf{D}_{\text {perf }}^{[0,3]}\left(E_{x}\right) .
$$

We study the natural specialization morphism

$$
s_{x}: \mathbf{L} f_{x}^{*} \mathbf{R} \widetilde{\Gamma}_{\text {str }}\left(G_{K}, M\right) \rightarrow \mathbf{R} \widetilde{\Gamma}_{\text {str }}\left(G_{K}, M_{x}\right)
$$

and in particular $\mathrm{H}^{2}\left(s_{x}\right)$.

It follows from the finiteness theorem and Proposition 2.6(2) that the formation of the strict ordinary local conditions commutes with $\mathbf{L} f_{x}^{*}$. We assume that $f_{x}^{*}\left(M^{I_{v}}\right) \stackrel{\sim}{\rightarrow}\left(M_{x}\right)^{I_{v}}$ so that the formation of the unramified local conditions commutes with $\mathbf{L} f_{x}^{*}$. Then the base-change theorem, situation (3) of Theorem 1.12, shows the morphism (3-3) to be an isomorphism, giving rise to a short exact sequence

$$
0 \rightarrow f_{x}^{*} \widetilde{\mathrm{H}}_{\mathrm{str}}^{2}\left(G_{K}, M\right) \stackrel{\mathrm{H}^{2}\left(s_{x}\right)}{\longrightarrow} \widetilde{\mathrm{H}}_{\mathrm{str}}^{2}\left(G_{K}, M_{x}\right) \rightarrow \operatorname{Tor}_{1}^{\mathbb{O} X}\left(\widetilde{\mathrm{H}}_{\mathrm{str}}^{3}\left(G_{K}, M\right), E_{x}\right) \rightarrow 0 .
$$

Thus, $\mathrm{H}^{2}\left(s_{x}\right)$ is an isomorphism precisely when $x$ avoids the support of the torsion in $\widetilde{\mathrm{H}}_{\mathrm{str}}^{3}\left(G_{K}, M\right)$.

On the other hand, we may relate $\widetilde{\mathrm{H}}_{\mathrm{str}}^{2}\left(G_{K}, M_{x}\right)$ to an extended Selmer group in degree 1 via duality. Namely, we equip $M_{x}^{*}(1)$ with the strict ordinary local conditions at $v \in S$ lying over $p$ built from the $\left(F_{v, x}^{\alpha_{v}}\right)^{\perp}$, and the unramified local conditions at $v \in S$ not dividing $p$, to construct the Selmer complex $\mathbf{R} \widetilde{\Gamma}_{\text {str }}\left(G_{K}, M_{x}^{*}(1)\right)$. The local conditions for $M_{x}$ and $M_{x}^{*}(1)$ are dual to one another. At places $v \in S$ lying over $p$, this follows from the duality of Galois cohomology of $(\varphi, \Gamma)$-modules 
contained in the finiteness theorem as previously mentioned. At places $v \in S$ not dividing $p$, there is a possible error in the integral self-duality of the unramified local conditions (coming from nontrivial Tamagawa numbers), but this contribution disappears after inverting $p$; see [Nekovár 2006, 7.6.7(iii)]. Thus, Theorem 1.16 gives

$$
\mathbf{R} \widetilde{\Gamma}_{\text {str }}\left(G_{K}, M_{x}\right) \cong \mathbf{R} \widetilde{\Gamma}_{\text {str }}\left(G, M_{x}^{*}(1)\right)^{*}[-3]
$$

in $\mathbf{D}_{\text {perf }}^{[0,3]}\left(E_{x}\right)$ and, in particular,

$$
\widetilde{\mathrm{H}}_{\mathrm{str}}^{2}\left(G_{K}, M_{x}\right) \cong \widetilde{\mathrm{H}}_{\mathrm{str}}^{1}\left(G_{K}, M_{x}^{*}(1)\right)^{*} .
$$

Now we relate the $\widetilde{\mathrm{H}}_{\mathrm{str}}^{1}\left(G_{K}, M_{x}^{*}(1)\right)$ to Bloch-Kato Selmer groups for $M_{x}^{*}(1)$ by computing the complexes $E_{v}=$ Cone $\left(i_{v}\right)$ appearing in the exact triangles (1-3) and (1-4). For $v \in S$ dividing $p$, one has $E_{v} \cong \mathbf{R} \Gamma\left(G_{v},\left(F_{v, x}^{\alpha_{v}}\right)^{*}(1)\right)$, whereas for $v \in S$ not dividing $p$ one has

$$
\mathrm{H}^{0} E_{v}=0 \quad \text { and } \quad \mathrm{H}^{1} E_{v} \cong \mathrm{H}_{\text {cont }}^{1}\left(I_{v}, M_{x}^{*}(1)\right)^{G_{v}} .
$$

Thus, one has an exact sequence

$$
\begin{aligned}
\cdots \rightarrow \bigoplus_{\substack{v \in S \\
\text { dividing } p}} \mathrm{H}^{0}\left(G_{v},\left(F_{v, x}^{\alpha_{v}}\right)^{*}(1)\right) \rightarrow \widetilde{\mathrm{H}}_{\mathrm{str}}^{1}\left(G_{K}, M_{x}^{*}(1)\right) \rightarrow \mathrm{H}_{\text {cont }}^{1}\left(G_{K, S}, M_{x}^{*}(1)\right) \\
\quad \rightarrow \bigoplus_{\substack{v \in S \\
\text { dividing } p}} \mathrm{H}^{1}\left(G_{v},\left(F_{x, v}^{\alpha_{v}}\right)^{*}(1)\right) \oplus \bigoplus_{\substack{v \in S \\
\text { not dividing } p}} \mathrm{H}_{\text {cont }}^{1}\left(I_{v}, M_{x}^{*}(1)\right)^{G_{v}} \rightarrow \cdots,
\end{aligned}
$$

and the image of $\widetilde{\mathrm{H}}_{\text {str }}^{1}\left(G_{K}, M_{x}^{*}(1)\right)$ in $\mathrm{H}_{\text {cont }}^{1}\left(G_{K, S}, M_{x}^{*}(1)\right)$ is identified to a subgroup cut out by local subspaces. For $v$ not dividing $p$, these are the usual unramified local subspaces, and for $v$ dividing $p$, these are strict ordinary local spaces in the sense of Section 3B. Therefore, assuming for each $v \in S$ dividing $p$ that the hypotheses of Proposition 3.7(3) for $\left.M_{x}^{*}(1)\right|_{G_{v}}$ hold, and in particular that each $\mathrm{H}^{0}\left(G_{v},\left(F_{x, v}^{\alpha_{v}}\right)^{*}(1)\right)=0$, we have $\widetilde{\mathrm{H}}_{\text {str }}^{1}\left(G_{K}, M_{x}^{*}(1)\right) \stackrel{\sim}{\rightarrow} \mathrm{H}_{\mathrm{f}}^{1}\left(G_{K}, M_{x}^{*}(1)\right)$, where the right-hand side is Bloch-Kato's Selmer group for $M_{x}^{*}(1)$.

Because the $\widetilde{\mathrm{H}}_{\text {str }}^{i}\left(G_{K}, M\right)$ are coherent sheaves on $X$, they have a reasonable structure theory. It follows that for $x$ varying over all points of $X$ with values in finite extensions $E_{x}$ of $E$, the number $\operatorname{dim}_{E_{x}} f_{x}^{*} \widetilde{\mathrm{H}}_{\text {str }}^{i}\left(G_{K}, M\right)$ is constant at its minimum value outside of a locally (i.e., over each affinoid) Zariski-closed proper subset. Further throwing away the support of the torsion in $\widetilde{\mathrm{H}}_{\mathrm{str}}^{3}\left(G_{K}, M\right)$, which only increases the resulting rank of $\widetilde{\mathrm{H}}_{\mathrm{str}}^{2}\left(G_{K}, M_{x}\right)$, we thus obtain the following:

Theorem 3.13. Let $M$ be a family of $G_{K, S}$-representations over $X$ that is nearly ordinary at each $v \in S$ dividing $p$ and for each $v \in S$ not dividing $p$ that $M^{I_{v}}$ is flat. Let $X_{0}$ be the set of points $x$ with values in finite extensions $E_{x}$ of $E$, for which 
- $f_{x}^{*}\left(M^{I_{v}}\right) \stackrel{\sim}{\rightarrow}\left(M_{x}\right)^{I_{v}}$ if $v$ does not divide $p$ and

- the hypotheses of Proposition 3.7(3) for $\left.M_{x}^{*}(1)\right|_{G_{v}}$ hold if $v$ divides $p$.

Then the Bloch-Kato Selmer groups of the $M_{x}^{*}(1)$ at the $x \in X_{0}$ have $E_{x}$-dimensions that are equal to their minimum except possibly on a locally Zariski-closed proper subset.

Remark 3.14. In the case where the family is over a reduced affinoid of dimension 1 , this statement is more or less equivalent to [Bellaïche 2012, Theorem 1].

\section{Acknowledgments}

Since this work grew out of investigations in my Ph.D. dissertation, I first thank my advisor, Barry Mazur, for his enthusiasm and encouragement. It was out of my desire to make rigorous many of his intuitions that I came upon this line of work. I benefited greatly from the works of Jan Nekovár and Ruochuan Liu, and I thank them heartily for their mathematical contributions. My recent collaboration with Kiran S. Kedlaya and Liang Xiao improved my understanding of many issues in this paper, and I am grateful to them. I am also indebted to Joël Bellaïche, Laurent Berger, Gaëtan Chenevier, Olivier Fouquet, Ben Howard, and Robert Pollack for numerous helpful conversations as well as the referee for suggesting several improvements in the exposition. I thank Tetsushi Ito for his repeated invitations to Japan, around which a great deal of progress on this project occurred, as well as Jan Nekovár for arranging my stay in Paris and l'Institut de Mathématiques de Jussieu for its hospitality. This work was supported by an NSF Graduate Research Fellowship.

\section{References}

[Amice and Vélu 1975] Y. Amice and J. Vélu, "Distributions $p$-adiques associées aux séries de Hecke", pp. 119-131 in Journées Arithmétiques de Bordeaux (Bordeaux, 1974), Astérisque 24/25, Société Mathématique de France, Paris, 1975. MR 51 \#12709 Zbl 0332.14010

[Andreatta and Iovita 2008] F. Andreatta and A. Iovita, "Global applications of relative $(\phi, \Gamma)-$ modules, I", pp. 339-420 in Représentations p-adiques de groupes p-adiques, I: Représentations galoisiennes et $(\phi, \Gamma)$-modules, Astérisque 319, Société Mathématique de France, Paris, 2008. MR 2010g:14020 Zbl 1163.11051

[Bellaïche 2012] J. Bellaïche, "Ranks of Selmer groups in an analytic family", Trans. Amer. Math. Soc 364 (2012), 4735-4761.

[Bellaïche and Chenevier 2009] J. Bellaïche and G. Chenevier, Families of Galois representations and Selmer groups, Astérisque 324, Société Mathématique de France, Paris, 2009. MR 2011m: 11105 Zbl 1192.11035

[Benois 2009] D. Benois, “On trivial zeroes of Perrin-Riou's $L$-functions”, preprint, 2009. arXiv 0906.2862

[Benois 2011] D. Benois, "A generalization of Greenberg's $\mathscr{L}$-invariant", Am. J. Math. 133:6 (2011), 1573-1632. MR 2863371 Zbl 5994421 
[Berger 2002] L. Berger, "Représentations $p$-adiques et équations différentielles", Invent. Math. 148:2 (2002), 219-284. MR 2004a:14022 Zbl 1113.14016

[Berger 2008] L. Berger, "Équations différentielles $p$-adiques et $(\phi, N)$-modules filtrés", pp. 13-38 in Représentations p-adiques de groupes p-adiques, I: Représentations galoisiennes et $(\phi, \Gamma)$-modules, Astérisque 319, Société Mathématique de France, Paris, 2008. MR 2010d:11056 Zbl 1168.11019

[Berger and Colmez 2008] L. Berger and P. Colmez, "Familles de représentations de de Rham et monodromie p-adique", pp. 303-337 in Représentations p-adiques de groupes p-adiques, I: Représentations galoisiennes et $(\phi, \Gamma)$-modules, Astérisque 319, Société Mathématique de France, Paris, 2008. MR 2010g:11091 Zbl 1168.11020

[Berthelot and Ogus 1978] P. Berthelot and A. Ogus, Notes on crystalline cohomology, Princeton University Press, 1978. MR 58 \#10908 Zbl 0383.14010

[Bloch and Kato 1990] S. Bloch and K. Kato, "L-functions and Tamagawa numbers of motives", pp. 333-400 in The Grothendieck Festschrift, vol. 1, edited by P. Cartier et al., Progr. Math. 86, Birkhäuser, Boston, MA, 1990. MR 92g:11063 Zbl 0768.14001

[Buzzard 2007] K. Buzzard, "Eigenvarieties", pp. 59-120 in L-functions and Galois representations (Durham, 2004), edited by D. Burns et al., London Math. Soc. Lecture Note Ser. 320, Cambridge University Press, 2007. MR 2010g:11076 Zbl 1230.11054

[Chenevier 2009] G. Chenevier, "Une application des variétés de Hecke des groupes unitaires", preprint, École Polytechnique, Paris, 2009, http://www.math.polytechnique.fr/ chenevier/articles/ famgal.pdf.

[Chenevier 2010] G. Chenevier, "Sur la densité des représentations cristallines du groupe de galois absolu de $\mathbb{Q} p$ ”, preprint, 2010. arXiv 1012.2852

[Colmez 2005] P. Colmez, "Série principale unitaire pour $\mathrm{GL}_{2}\left(\mathbb{Q}_{p}\right)$ et représentations triangulines de dimension 2", preprint, 2005, http://www.math.jussieu.fr/ colmez/trianguline.pdf.

[Colmez 2008] P. Colmez, "Représentations triangulines de dimension 2", pp. 213-258 in Représentations p-adiques de groupes p-adiques, I: Représentations galoisiennes et $(\phi, \Gamma)$-modules, edited by L. Berger et al., Astérisque 319, Société Mathématique de France, Paris, 2008. MR 2010f:11173 Zbl 1168.11022

[Colmez 2010] P. Colmez, "La série principale unitaire de $\mathrm{GL}_{2}\left(\mathbf{Q}_{p}\right)$ ", pp. 213-262 in Représentations p-adiques de groupes p-adiques, II: Représentations de $\mathrm{GL}_{2}\left(\mathbf{Q}_{p}\right)$ et $(\phi, \Gamma)$-modules], edited by L. Berger et al., Astérisque 330, Société Mathématique de France, Paris, 2010. MR 2011g:22026 Zbl 1242.11095

[Colmez and Fontaine 2000] P. Colmez and J.-M. Fontaine, "Construction des représentations $p$ adiques semi-stables”, Invent. Math. 140:1 (2000), 1-43. MR 2001g:11184 Zbl 1010.14004

[Flach 1990] M. Flach, “A generalisation of the Cassels-Tate pairing”, J. Reine Angew. Math. 412 (1990), 113-127. MR 92b:11037 Zbl 0711.14001

[Fontaine and Perrin-Riou 1994] J.-M. Fontaine and B. Perrin-Riou, "Autour des conjectures de Bloch et Kato: cohomologie galoisienne et valeurs de fonctions L", pp. 599-706 in Motives (Seattle, WA, 1991), edited by U. Jannsen et al., Proc. Sympos. Pure Math. 55, American Mathematical Society, Providence, RI, 1994. MR 95j:11046 Zbl 0821.14013

[Fukaya and Kato 2006] T. Fukaya and K. Kato, "A formulation of conjectures on p-adic zeta functions in noncommutative Iwasawa theory", pp. 1-85 in Proceedings of the St. Petersburg Mathematical Society, vol. 12, edited by N. N. Uraltseva, Amer. Math. Soc. Transl. Ser. 2 219, American Mathematical Society, Providence, RI, 2006. MR 2007k:11200 Zbl 1238.11105 
[Greenberg 1989] R. Greenberg, "Iwasawa theory for p-adic representations", pp. 97-137 in Algebraic number theory, edited by J. Coates et al., Adv. Stud. Pure Math. 17, Academic Press, Boston, MA, 1989. MR 92c:11116 Zbl 0739.11045

[Greenberg 1994a] R. Greenberg, "Iwasawa theory and p-adic deformations of motives", pp. 193-223 in Motives (Seattle, WA, 1991), edited by U. Jannsen et al., Proc. Sympos. Pure Math. 55, American Mathematical Society, Providence, RI, 1994. MR 95i:11053 Zbl 0819.11046

[Greenberg 1994b] R. Greenberg, "Trivial zeros of p-adic L-functions", pp. 149-174 in p-adic monodromy and the Birch and Swinnerton-Dyer conjecture (Boston, MA, 1991), edited by B. Mazur and G. Stevens, Contemp. Math. 165, American Mathematical Society, Providence, RI, 1994. MR 95h:11063 Zbl 0838.11070

[Hellmann 2010] E. Hellmann, "On arithmetic families of filtered phi-modules and crystalline representations”, preprint, 2010. arXiv 1010.4577

[Hellmann 2012a] E. Hellmann, "Families of $p$-adic Galois representations and $(\varphi, \Gamma)$-modules", preprint, 2012. arXiv 1202.3413

[Hellmann 2012b] E. Hellmann, "Families of trianguline representations and finite slope subspaces", preprint, 2012. arXiv 1202.4408

[Herr 1998] L. Herr, "Sur la cohomologie galoisienne des corps p-adiques", Bull. Soc. Math. France 126:4 (1998), 563-600. MR 2000m:11118 Zbl 0967.11050

[de Jong 1995] A. J. de Jong, "Crystalline Dieudonné module theory via formal and rigid geometry", Inst. Hautes Études Sci. Publ. Math. 82 (1995), 5-96. MR 97f:14047 Zbl 0864.14009

[Kato 2004] K. Kato, " $p$-adic Hodge theory and values of zeta functions of modular forms”, pp. 117-290 in Cohomologies p-adiques et applications arithmétiques, vol. 3, edited by P. Berthelot et al., Astérisque 295, 2004. MR 2006b:11051 Zbl 1142.11336

[Kedlaya 2008] K. S. Kedlaya, "Slope filtrations for relative Frobenius", pp. 259-301 in Représentations p-adiques de groupes p-adiques, I: Représentations galoisiennes et $(\phi, \Gamma)$-modules, edited by L. Berger et al., Astérisque 319, 2008. MR 2010c:14024 Zbl 1168.11053

[Kedlaya and Liu 2010] K. Kedlaya and R. Liu, "On families of $\phi$, $\Gamma$-modules", Algebra Number Theory 4:7 (2010), 943-967. MR 2012h:11081 Zbl 05852025

[Kedlaya et al. 2012] S. Kedlaya, Kiran, J. Pottharst, and L. Xiao, "Cohomology of arithmetic families of $(\varphi, \Gamma)$-modules", preprint, 2012. arXiv 1203.5718

[Kisin 2003] M. Kisin, "Overconvergent modular forms and the Fontaine-Mazur conjecture", Invent. Math. 153:2 (2003), 373-454. MR 2004f:11053 Zbl 1045.11029

[Liu 2007] R. Liu, "Cohomology and duality for $(\phi, \Gamma)$-modules over the Robba ring”, Int. Math. Res. Not. 2007:3 (2007), Art. ID rnm150. MR 2009e:11222 Zbl 1248.11093

[Liu 2012] R. Liu, “Triangulation of refined families”, preprint, 2012. arXiv 1202.2188

[Matsumura 1989] H. Matsumura, Commutative ring theory, 2nd ed., Cambridge Studies in Advanced Mathematics 8, Cambridge University Press, 1989. MR 90i:13001 Zbl 0666.13002

[Mazur 1972] B. Mazur, "Rational points of abelian varieties with values in towers of number fields", Invent. Math. 18 (1972), 183-266. MR 56 \#3020 Zbl 0245.14015

[Mazur et al. 1986] B. Mazur, J. Tate, and J. Teitelbaum, "On p-adic analogues of the conjectures of Birch and Swinnerton-Dyer”, Invent. Math. 84:1 (1986), 1-48. MR 87e:11076 Zbl 0699.14028

[Nekovář 2006] J. Nekovář, Selmer complexes, Astérisque 310, 2006. MR 2009c:11176 Zbl 1211 11120

[Neukirch et al. 2008] J. Neukirch, A. Schmidt, and K. Wingberg, Cohomology of number fields, 2nd ed., Grundlehren Math. Wiss. 323, Springer, Berlin, 2008. MR 2008m:11223 Zbl 1136.11001 
[Perrin-Riou 1994a] B. Perrin-Riou, "Représentations p-adiques ordinaires", pp. 185-220 in Périodes p-adiques (Bures-sur-Yvette, 1988), Astérisque 223, 1994. MR 96h:11063 Zbl 1043.11532

[Perrin-Riou 1994b] B. Perrin-Riou, "Théorie d'Iwasawa des représentations $p$-adiques sur un corps local”, Invent. Math. 115:1 (1994), 81-161. MR 95c:11082 Zbl 0838.11071

[Perrin-Riou 2000] B. Perrin-Riou, p-adic L-functions and p-adic representations, SMF/AMS Texts and Monographs 3, American Mathematical Society, Providence, RI, 2000. MR 2000k:11077 Zbl 0988.11055

[Pottharst 2012] J. Pottharst, "Cyclotomic Iwasawa theory of motives", preprint, 2012, http:// math.bu.edu/people/potthars/writings/cyc.pdf.

[Schneider 1987] P. Schneider, "Arithmetic of formal groups and applications, I: Universal norm subgroups", Invent. Math. 87:3 (1987), 587-602. MR 88e:11044 Zbl 0608.14034

[Schneider and Teitelbaum 2003] P. Schneider and J. Teitelbaum, "Algebras of $p$-adic distributions and admissible representations", Invent. Math. 153:1 (2003), 145-196. MR 2004g:22015 Zbl 1028.11070

[Višik 1976] M. M. Višik, "Nonarchimedean measures associated with Dirichlet series", Mat. Sb. (N.S.) 99(141):2 (1976), 248-260. In Russian; translated in Math. USSR Sb. 28:2 (1976), 216-228. MR 54 \#243 Zbl 0358.14014

Communicated by John Henry Coates

Received 2011-08-17 Revised 2012-07-28 Accepted 2012-12-17

potthars@bu.edu

Department of Mathematics and Statistics, Boston University, 111 Cummington Mall, Boston, MA, 02215, United States 


\section{Algebra \& Number Theory}

msp.org/ant

\section{EDITORS}

MANAGING EDITOR

Bjorn Poonen

Massachusetts Institute of Technology

Cambridge, USA

\author{
EDITORIAL BOARD CHAIR \\ David Eisenbud \\ University of California \\ Berkeley, USA
}

\section{BOARD OF EDITORS}

Georgia Benkart

Dave Benson

Richard E. Borcherds

John H. Coates

J-L. Colliot-Thélène

Brian D. Conrad

Hélène Esnault

Hubert Flenner

Edward Frenkel

Andrew Granville

Joseph Gubeladze

Roger Heath-Brown

Ehud Hrushovski

Craig Huneke

Mikhail Kapranov

Yujiro Kawamata

János Kollár

Yuri Manin

Barry Mazur

Philippe Michel
University of Wisconsin, Madison, USA

University of Aberdeen, Scotland

University of California, Berkeley, USA

University of Cambridge, UK

CNRS, Université Paris-Sud, France

University of Michigan, USA

Freie Universität Berlin, Germany

Ruhr-Universität, Germany

University of California, Berkeley, USA

Université de Montréal, Canada

San Francisco State University, USA

Oxford University, UK

Hebrew University, Israel

University of Virginia, USA

Yale University, USA

University of Tokyo, Japan

Princeton University, USA

Northwestern University, USA

Harvard University, USA

École Polytechnique Fédérale de Lausanne
Susan Montgomery

Shigefumi Mori

Raman Parimala

Jonathan Pila

Victor Reiner

Karl Rubin

Peter Sarnak

Joseph H. Silverman

Michael Singer

Vasudevan Srinivas

J. Toby Stafford

Bernd Sturmfels

Richard Taylor

Ravi Vakil

Michel van den Bergh

Marie-France Vignéras

Kei-Ichi Watanabe

Efim Zelmanov

Shou-Wu Zhang
University of Southern California, USA

RIMS, Kyoto University, Japan

Emory University, USA

University of Oxford, UK

University of Minnesota, USA

University of California, Irvine, USA

Princeton University, USA

Brown University, USA

North Carolina State University, USA

Tata Inst. of Fund. Research, India

University of Michigan, USA

University of California, Berkeley, USA

Harvard University, USA

Stanford University, USA

Hasselt University, Belgium

Université Paris VII, France

Nihon University, Japan

University of California, San Diego, USA

Princeton University, USA

PRODUCTION

production@msp.org

Silvio Levy, Scientific Editor

See inside back cover or msp.org/ant for submission instructions.

The subscription price for 2013 is US $\$ 200 /$ year for the electronic version, and $\$ 350 /$ year $(+\$ 40$, if shipping outside the US) for print and electronic. Subscriptions, requests for back issues and changes of subscribers address should be sent to MSP.

Algebra \& Number Theory (ISSN 1944-7833 electronic, 1937-0652 printed) at Mathematical Sciences Publishers, 798 Evans Hall \#3840, c/o University of California, Berkeley, CA 94720-3840 is published continuously online. Periodical rate postage paid at Berkeley, CA 94704, and additional mailing offices.

ANT peer review and production are managed by EditFLOW ${ }^{\circledR}$ from Mathematical Sciences Publishers.

\section{PUBLISHED BY}

- mathematical sciences publishers

nonprofit scientific publishing

http://msp.org/

(C) 2013 Mathematical Sciences Publishers 


\section{Algebra \& Number Theory}

$\begin{array}{lll}\text { Volume } 7 & \text { No. } 7 \quad 2013\end{array}$

Weil representation and transfer factor

TERUJI THOMAS

Analytic families of finite-slope Selmer groups

1571

JONATHAN POTTHARST

Multiplicative excellent families of elliptic surfaces of type $E_{7}$ or $E_{8}$

ABHINAV KUMAR and TETSUJI SHIODA

Cohomological invariants of algebraic tori

SAM Blinstein and AleXANDer MerkurJeV

On abstract representations of the groups of rational points of algebraic groups and their 1685 deformations

IGOR A. RAPINCHUK

Betti diagrams from graphs

AlEXANDER ENGSTRÖM and MATTHEW T. STAMPS

Hopf monoids from class functions on unitriangular matrices

Marcelo Aguiar, NANTEl Bergeron and Nathaniel Thiem 